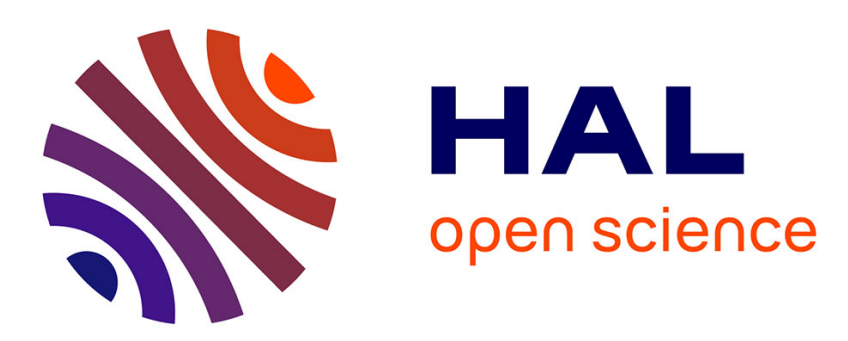

\title{
Transcrystallinity versus spherulitic crystallization in polyamide 66: An experimental study
}

Lionel Freire, Christelle Combeaud, Gabriel Monge, Noëlle Billon, Jean-Marc

Haudin

\section{- To cite this version: \\ Lionel Freire, Christelle Combeaud, Gabriel Monge, Noëlle Billon, Jean-Marc Haudin. Transcrys- tallinity versus spherulitic crystallization in polyamide 66: An experimental study. Polymer Crystal- lization, 2019, 2 (1), pp.e10028. 10.1002/pcr2.10028 . hal-01987926}

\section{HAL Id: hal-01987926 \\ https://hal.science/hal-01987926}

Submitted on 26 Mar 2019

HAL is a multi-disciplinary open access archive for the deposit and dissemination of scientific research documents, whether they are published or not. The documents may come from teaching and research institutions in France or abroad, or from public or private research centers.
L'archive ouverte pluridisciplinaire HAL, est destinée au dépôt et à la diffusion de documents scientifiques de niveau recherche, publiés ou non, émanant des établissements d'enseignement et de recherche français ou étrangers, des laboratoires publics ou privés. 


\section{Polymer Crystallization}

\section{Transcrystalliny vs. spherulitic crystallization in polyamide 66. An experimental study --Manuscript Draft--}

\begin{tabular}{|c|c|}
\hline Manuscript Number: & pxl.20180030R1 \\
\hline Full Title: & Transcrystalliny vs. spherulitic crystallization in polyamide 66 . An experimental study \\
\hline Article Type: & Full Papers \\
\hline Keywords: & polyamide 66 , crystallization, spherulite, transcrystallinity, growth rate \\
\hline Corresponding Author: & $\begin{array}{l}\text { Jean-Marc HAUDIN } \\
\text { Mines ParisTech } \\
\text { Sophia -Antipolis, Alpes-Maritimes FRANCE }\end{array}$ \\
\hline Corresponding Author's Institution: & Mines ParisTech \\
\hline First Author: & Jean-Marc HAUDIN \\
\hline \multirow[t]{5}{*}{ Order of Authors: } & Jean-Marc HAUDIN \\
\hline & Lionel Freire \\
\hline & Christelle COMBEAUD \\
\hline & Gabriel MONGE \\
\hline & Noëlle BILLON \\
\hline Abstract: & $\begin{array}{l}\text { The crystallization of three polyamides } 66 \text { has been studied by optical microscopy and } \\
\text { differential scanning calorimetry (DSC). Non-isothermal crystallization kinetics have } \\
\text { been analyzed using Ozawa's equation. Two polymers have close behaviors, with a } \\
\text { crystallization mainly spherulitic. The third one, which has the highest molar mass, is } \\
\text { subject to intense transcrystallinity, which disturbs the crystallization kinetics (shoulder } \\
\text { on the DSC curves, low Avrami exponent). For this polymer, original methods based on } \\
\text { a detailed analysis of transcrystallinity have been applied to measure crystal growth } \\
\text { rate and to determine the "intrinsic" crystallization kinetics, i.e., not polluted by } \\
\text { transcrystallinity }\end{array}$ \\
\hline \multicolumn{2}{|l|}{ Additional Information: } \\
\hline Question & Response \\
\hline $\begin{array}{l}\text { Please submit a plain text version of your } \\
\text { cover letter here. } \\
\text { Please note, if you are submitting a } \\
\text { revision of your manuscript, there is an } \\
\text { opportunity for you to provide your } \\
\text { responses to the reviewers later; please } \\
\text { do not add them to the cover letter. }\end{array}$ & $\begin{array}{l}\text { Dear Editor, } \\
\text { Please find enclosed the revised version of our paper entitled "Transcrystalliny vs. } \\
\text { spherulitic crystallization in polyamide } 66 \text {. An experimental study", which is submitted } \\
\text { for publication in Polymer Crystallization. } \\
\text { We thank the reviewer for his positive evaluation and his very interesting and subtle } \\
\text { remarks, which essentially concern a possible role of branching. These remarks have } \\
\text { been completely taken into account: } \\
\text { 1. We added new comments for Fig 9, discussing the point put forward by the reviewer: } \\
\text { "A more detailed comparison between PA66-2 and PA66-6 is difficult because the } \\
\text { thicknesses of the films are not exactly the same, and as will be seen for PA66-4, an } \\
\text { increase in the sample thickness increases core crystallization. Nevertheless, if we } \\
\text { compare the size of spherulites in PA66-2 cooled at } 50^{\circ} \mathrm{C} / \text { min and in PA66- } 6 \text { cooled at } \\
100^{\circ} \mathrm{C} / \mathrm{min} \text {, we should have smaller spherulites in PA66-6 sample if crystallization in } \\
\text { these two grades was exactly the same, which is not the case. Therefore, branching } \\
\text { might have an effect on bulk nucleation, hence on transcrystallinity, but this should be } \\
\text { confirmed by further investigation. This would be consistent with the slower spherulitic } \\
\text { growth and volume crystallization that have been reported in the literature for branched } \\
\text { polyamide [25]". }\end{array}$ \\
\hline
\end{tabular}


and added a reference corresponding to the remark of the reviewer: [25] S. Acierno, P. van Puyvelde, Polymer 2005, 46, 10331.

2. A sentence in the conclusion has been modified: "It appears that branching does not play any detectable role on crystallization temperatures and overall kinetics, a possible role on nucleation rate could be studied in further investigations".

The changes to the manuscript are marked in red.

Please consider the revised manuscript for publication in Polymer Crystallization.

Best regards.

Prof. Jean-Marc HAUDIN

August 23, 2018 


\title{
Transcrystalliny vs. spherulitic crystallization in polyamide 66. An experimental study
}

\author{
Lionel Freire | Christelle Combeaud | Gabriel Monge | Noëlle Billon | Jean- \\ Marc Haudin
}

MINES ParisTech, PSL Research University, CEMEF - Centre de Mise en Forme des Matériaux, UMR CNRS 7635, Sophia Antipolis, France

Correspondence

Jean-Marc Haudin, MINES ParisTech, PSL Research University, CEMEF - Centre de Mise en Forme des Matériaux, CNRS UMR 7635, CS 10207, 1 rue Claude Daunesse, 06904 Sophia Antipolis Cedex, France.

E-mail address: jean-marc.haudin@mines-paristech.fr

\begin{abstract}
The crystallization of three polyamides 66 has been studied by optical microscopy and differential scanning calorimetry (DSC). Non-isothermal crystallization kinetics have been analyzed using Ozawa's equation. Two polymers have close behaviors, with a crystallization mainly spherulitic. The third one, which has the highest molar mass, is subject to intense transcrystallinity, which disturbs the crystallization kinetics (shoulder on the DSC curves, low Avrami exponent). For this polymer, original methods based on a detailed analysis of transcrystallinity have been applied to measure crystal growth rate and to determine the “intrinsic" crystallization kinetics, i.e., not polluted by transcrystallinity.
\end{abstract}

\section{KEYWORDS}

polyamide 66 , crystallization, spherulite, transcrystallinity, growth rate 


\section{1 | INTRODUCTION}

Like for other polyamides, crystalline structure, morphology and resulting properties of polyamide 66 (PA66) are strongly influenced by hydrogen bonding. A first consequence is the sensitivity of this polymer to moisture, which imposes to control the water content in order to obtain reliable and reproducible results.

Crystalline structures of PA66 consist of hydrogen-bonded sheets. Hydrogen bonds between the $\mathrm{N}-\mathrm{H}$ and $\mathrm{C}-\mathrm{O}$ groups on neighboring chains result in the formation of sheets of molecular segments, in which the intermolecular forces are considerably larger than the forces between segments in neighboring sheets. The relative arrangement of these sheets leads to two possible crystalline structures: $\alpha$ triclinic and $\beta$ monoclinic, in which the chain conformation is the planar zigzag. ${ }^{[1]}$ A reversible phase transition, from the triclinic $\alpha$-phase to the pseudohexagonal $\gamma$-structure, occurs at the Brill transition temperature $T_{B .}{ }^{[2]}$ It has been found that $T_{B}$ depends strongly on the crystallization conditions, e.g., with values of 160$225^{\circ} \mathrm{C}$ for crystallizations from the melt between 196 and $260^{\circ} \mathrm{C} .{ }^{[3]}$ In the $\gamma$ phase, the chains are not in the planar zigzag conformation, with a distortion at the amide group level. As a consequence, the periodicity along the chain axis is shorter. ${ }^{[4]}$

A careful analysis of small angle X-ray scattering results (SAXS) has pointed out an unusual behavior: ${ }^{[5]}$ the crystallization of lamellae with an approximately constant thickness, composed of a fully ordered core and a somewhat disordered inner layer, which is absorbed into the lengthening crystal core during long-term crystallization or annealing. This behavior is significantly different from that of non-polar polymers like linear polyethylene.

Spherulitic crystallization in PA66 has been considered by several authors. ${ }^{[6-9]}$ Four kinds of spherulites have been described by Magill ${ }^{[7]}$ according to the crystallization conditions: positive spherulites ringed or not, negative spherulites, spherulitic aggregates, nonbirefringent spherulites. Lovinger ${ }^{[9]}$ has found that in positive spherulites, the $\boldsymbol{a}$-axis of the unit cell is parallel to the spherulite radius. The hydrogen bonding is in $(\boldsymbol{a}, \boldsymbol{c})$ planes in the direction of crystal growth $\boldsymbol{a}, \boldsymbol{c}$ being the chain axis. The chains are folding over along the radius of the spherulite, which causes the positive birefringence. Growth rate measurements are given in a number of papers. ${ }^{[10-15]}$ Only the most recent ones tried to interpret the growth rate data in 
terms of the growth regimes predicted by Hoffman-Lauritzen's theory. ${ }^{[16]}$ For isothermal conclude that crystallization occurred in regime I. Lee and Phillips ${ }^{[15]}$ found a II-I regime transition at $239{ }^{\circ} \mathrm{C}$. In spite of this result, they estimated that Hoffman-Lauritzen's theory was not appropriate to describe crystallization in positive spherulites, from the analysis of chain folding done by Lovinger ${ }^{[9]}$ (see above).

In previous studies of PA66 in our laboratory, ${ }^{[17,18]}$ a lack of reproducibility was observed in crystallization experiments performed by differential scanning calorimetry (DSC). Typically, considering four specimens prepared with the same protocol, the shape of the crystallization curves varied significantly from one specimen to another: three curves exhibited a more or less pronounced shoulder, whereas the fourth one showed a single crystallization peak. This behavior could not be related to drying conditions, nor to the annealing treatment before crystallization. In addition, significant differences were also observed according to the origin of the pellets provided by the same manufacturer. It was then suggested that the differences observed resulted from differences in nucleation density. If the number of "natural" volume nuclei is too low, the polymer is very sensitive to heterogeneous nucleation at the surface of any foreign body (impurity, surface generating transcrystallinity, ...), which may occur in a more or less erratic way. As a consequence of the abovementioned experiments, it appeared that the competition between surface and bulk nucleation could be appropriately studied through crystallizations of thin polymer films in contact with aluminium pans surfaces in a DSC apparatus. It was shown that the thermograms with a shoulder corresponded to important transcrystalline regions and coarse spherulites at the specimen core. Conversely, the thermograms which exhibited a single peak were associated with thin transcrystalline regions and a large number of small spherulites at the core. Consequently, the shoulder was correlated to transcrystallinity and to the competition between surface and volume nucleation. This assumption was checked by experiments on nucleated PA66: when a nucleating agent, promoting volume nucleation, is added to the polymer, the shoulder-shaped peak becomes a single one, and its maximum is shifted towards higher temperature. ${ }^{[18]}$ This experimental analysis was completed by numerical simulation. ${ }^{[19]}$ At the very first steps of the transformation, the transformed volume consists of distinct semi-spherical spherulites. As the spherulites grow, their growth is progressively limited to directions more or less perpendicular to the surfaces. Step by step, the transformed volume becomes more "compact" and finally, the transformation is achieved by the propagation of two continuous fronts. This change from 
half-spheres to continuous fronts is responsible for the shoulder observed in the DSC traces. This analysis definitely confirms that the shoulder at the beginning of the DSC peaks can be unambiguously related to the transcrystalline zones.

A series of experiments was performed with films of high density polyethylene (HDPE) of thicknesses ranging from 192 to $865 \mu \mathrm{m} .{ }^{[20]}$ All the samples had important transcrystalline zones at their surfaces. Thin samples were almost completely overlapped by transcrystalline regions. Medium-thickness samples contained more volume spherulites, but one row of spherulites at the most appeared in their mid-plane. Thick samples, for their part, contained two larger spherulites in the part of the thickness which was not overlapped by transcrystalline zones. The total thickness of the transcrystalline zones and the maximum diameter of the bulk spherulites both increased up to a maximum value of about $350 \mu \mathrm{m}$, which was reached for the same sample thickness. These results show that in thin samples the transcrystalline thickness is mainly limited by the sample thickness. The higher the thickness, the more important the transcrystalline zones up to a certain value, because in thick samples volume spherulites are more numerous and stop the development of transcrystallinity. From a careful analysis of the experimental data, it was also possible to derive original methods, which gave access to crystallization parameters (number of nuclei per unit surface, growth rate), and allowed to determine the crystallisation kinetics of the polymer itself, i.e., not disturbed by transcrystallinity. ${ }^{[21]}$

The first objective of this paper is to study the occurrence of transcrystallinity in three well-defined PA66 grades by combining optical microscopy and DSC. The influence of transcrystallinity on overall crystallization kinetics is analyzed. In the case of intense transcrystallinity, the original method proposed in Reference [21] for HDPE growth-rate measurements is applied to PA66. The results are compared to those obtained by other methods and to literature data. Finally, the "intrinsic" crystallization kinetics of the polymer, i.e., not disturbed by transcrystallinity, is determined.

\section{2 | EXPERIMENTAL}

\subsection{Materials and specimen preparation}




\begin{tabular}{|c|c|c|}
\hline Grade & $M_{n}(\mathrm{~g} / \mathrm{mol})$ & Structure \\
\hline PA66-2 & 24040 & linear \\
\hline PA66-4 & 38690 & linear \\
\hline PA66-6 & 17930 & branched \\
\hline
\end{tabular}

As PA66 is very hygroscopic, it is important to control sample preparation (vacuum drying and storage). Films of polymer were prepared from pellets melted between two glass slides in a FP82 hot stage (Mettler-Toledo, Viroflay, France), heated at $275^{\circ} \mathrm{C}$ and then cooled down to room temperature. The glass slides were treated with dichloro(dimethyl)silane to avoid adhesion of polymer on glass. Thickness of polymer films ranged between 100 and $200 \mu \mathrm{m}$. A special set of PA66-4 films with thicknesses ranging from 100 to $600 \mu \mathrm{m}$ was prepared for the study of transcrystallinity. For DSC experiments, films were cut into pieces to be inserted in DSC pans. Both films and pieces of films were vacuum dried at $90^{\circ} \mathrm{C}$ under $200 \mathrm{mbar}$ during a night, and then stored in a dry atmosphere prior to optical microscopy or DSC experiments.

\section{2 | DSC measurements}

DSC analyses were carried out using a DSC 8500 apparatus (PerkinElmer, Waltham, USA) to study crystallization at constant cooling rates up to $700^{\circ} \mathrm{C} / \mathrm{min}$. The calorimeter was calibrated using indium and tin in the heating mode, from $1^{\circ} \mathrm{C} / \mathrm{min}$ to $700^{\circ} \mathrm{C} / \mathrm{min}$. Therefore, calibration files were available for each cooling rate studied. Dried sample was heated at $10^{\circ} \mathrm{C} / \mathrm{min}$ up to $300^{\circ} \mathrm{C}$ and remained $15 \mathrm{~s}$ at this temperature in order to erase thermal history without polymer degradation. Then, it was cooled down at cooling rates ranging from 1 to $700^{\circ} \mathrm{C} / \mathrm{min}$. Each sample was used for only one thermal cycle in order to avoid cumulative degradation. 


\subsection{Optical microscopy}

Isothermal crystallizations were performed on the FP82 hot stage, between glass slides treated with dichloro(dimethyl)silane. They were observed between crossed polarizers, with or without gypsum plate, using a Leica DMRX microscope (Leica-Microsystèmes, Nanterre, France) with an attached CCD camera. As for DSC, dried films, prepared as indicated above, were used only once for a given experiment. The thermal protocol was the following: (i) holding $3 \mathrm{~min}$ à $100^{\circ} \mathrm{C}$; (ii) heating at $10^{\circ} \mathrm{C} / \mathrm{min}$ to $300^{\circ} \mathrm{C}$ to erase previous history, with no holding, which prevents from degradation; (iii) cooling at $10^{\circ} \mathrm{C} / \mathrm{min}$ to the crystallization temperature and following isothermal crystallization; (iv) cooling to room temperature. Isothermal crystallizations were studied between 240 and $253^{\circ} \mathrm{C}$. Below $240^{\circ} \mathrm{C}$, crystallization began before reaching the isothermal plateau. Above $253^{\circ} \mathrm{C}$, crystallization kinetics was too slow.

Microscopic observations were also done on sections (thickness: tenth of micrometers) cut from pellets, isothermally crystallized films or DSC specimens using a LKB Ultrotome 4800 microtome, equipped with a glass knife.

\section{3 | RESULTS AND DISCUSSION}

\section{1 | Preliminary investigation}

Isothermal crystallizations between treated glass slides were performed between 240 and $253^{\circ} \mathrm{C}$. Figure 1 shows the morphologies in PA66-4 after the isothermal crystallization at $244^{\circ} \mathrm{C}$. At first glance, we observe positive spherulites with a radius between 10 and $50 \mu \mathrm{m}$. Some hyperbolic boundaries confirm the sporadic in time character of nucleation. This 2D observation is fallacious, because the examination of a cross section of the crystallized film reveals in fact a morphology which is almost fully transcrystalline (Figure 2b). Conversely, PA66-2 (Figure 2a) and PA66-6 (Figure 2c) films crystallized in the same conditions contain a homogeneous distribution of small positive spherulites, with no surface nucleation. 
FIGURE 1 Observation by optical microscopy of the morphologies in PA66-4 after

isothermal crystallization at $244^{\circ} \mathrm{C}$

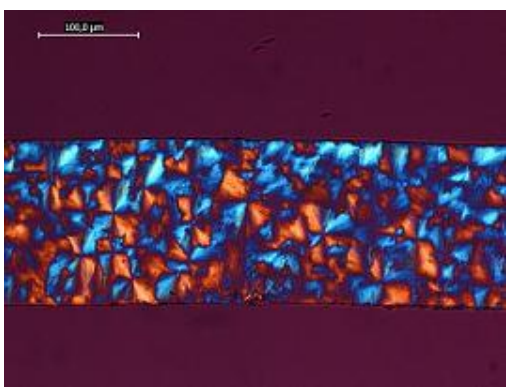

a

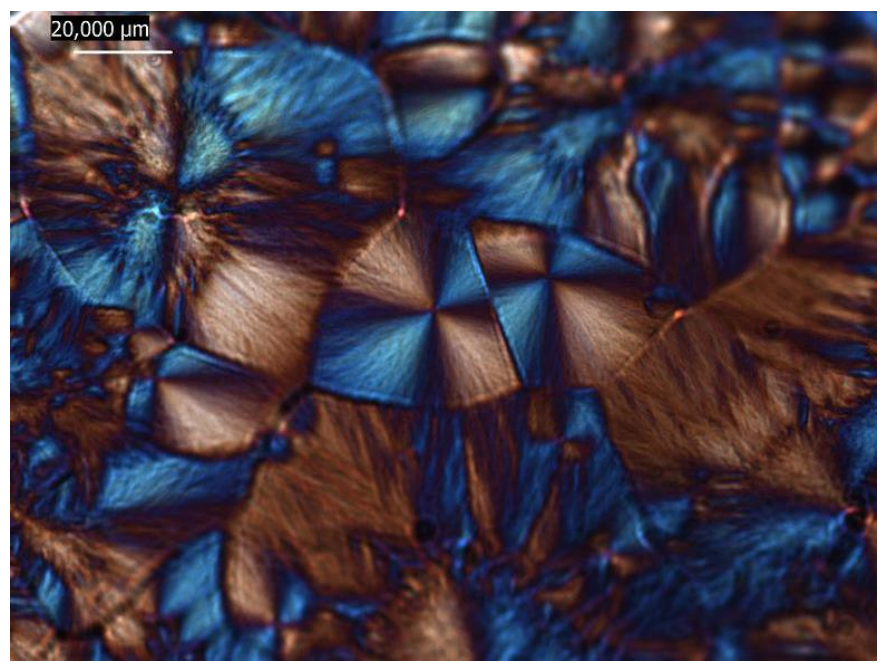

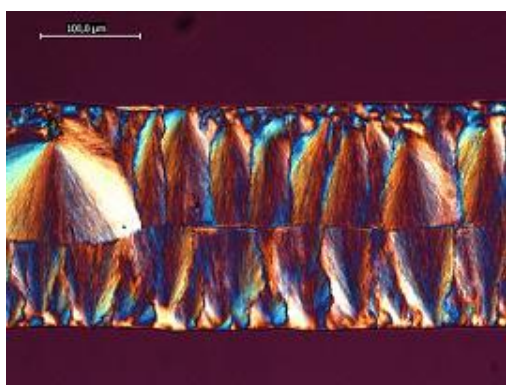

b

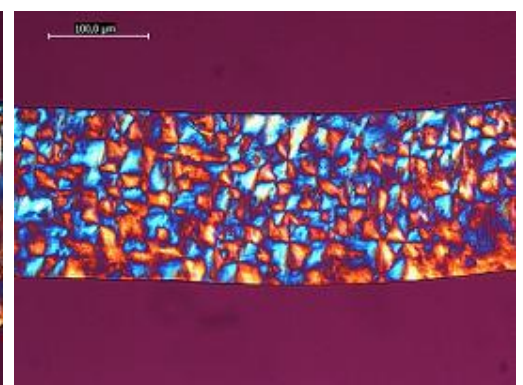

C

FIGURE 2 Observation of the cross sections of films after isothermal crystallization at 244 C: a) PA66-2; b) PA66-4; c) PA66-6. Scale bar: $100 \mu \mathrm{m}$

To avoid parasitic effects likely to occur in crystallization from thin films, e.g., flow or surface effects, crystallizations were carried out directly from pellets put on a glass substrate. The pellets were heated up to $300^{\circ} \mathrm{C}$, and then slowly cooled down to room temperature, the oven being turned off. Once more, PA66-2 and PA66-6 (Figures 3a and 3c) contain a homogeneous population of small spherulites. The microstructure of PA66-4 is heterogeneous (Figure 3b): (i) small entities at the free surface resulting from a population of nuclei coming from previous history or from interfacial stresses due to curvature; (ii) coarse spherulites at the core, which were able to develop during the slow cooling; (iii) a transcrystalline region at the contact between polymer and substrate. 


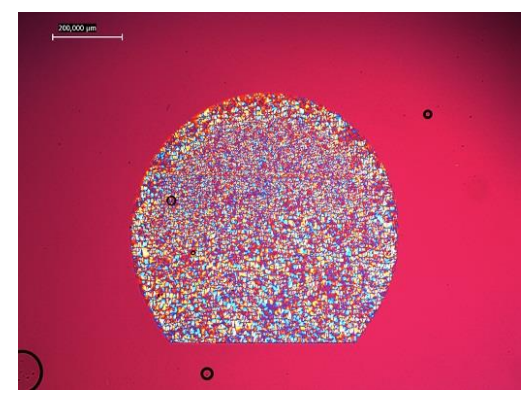

a

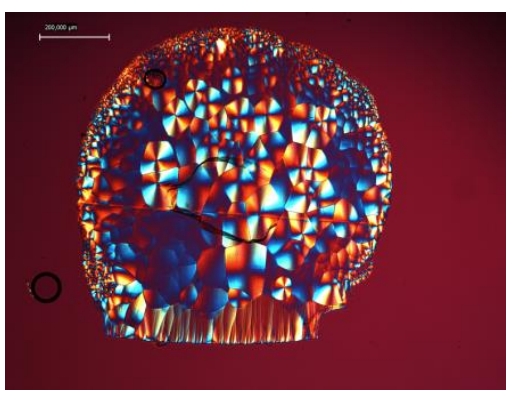

b

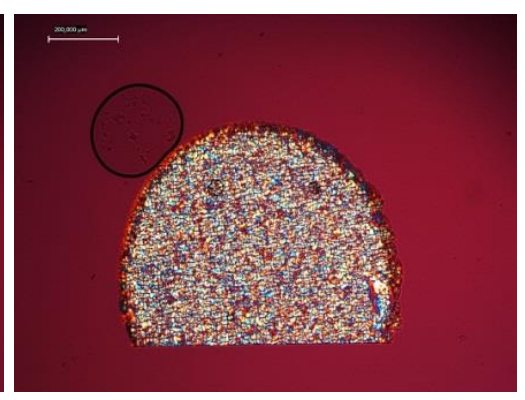

c

FIGURE 3 Observation of the cross sections of pellets after crystallization during a slow cooling: a) PA66-2; b) PA66-4; c) PA66-6. Scale bar: $200 \mu \mathrm{m}$

To sum up, volume nucleation seems to be easy in PA66-2 and PA66-6, whose behavior is similar. The density of volume nuclei is much lower in PA66-4, perhaps because of its higher molar mass. Consequently, when surfaces are present, competition between volume nucleation and surface occurs, which can lead to transcrystallinity. After this first qualitative approach, further analysis of the differences between the three polymers was done comparing their non-isothermal crystallization kinetics.

\section{2 | Non-isothermal crystallization}

\subsubsection{DSC measurements}

Figure 4 presents the DSC traces obtained for crystallization at various cooling rates. PA66-2 and PA66-6 exhibit a single almost symmetrical peak, with a tail possibly due to secondary crystallization. For PA66-4, crystallization curves show a shoulder at the beginning of solidification due to transcrystallinity phenomena, as suggested by literature data ${ }^{[18]}$ and isothermal crystallization results $(\S 3.1)$.

Two parameters were extracted from DSC data (Figure 5): (i) the crystallization temperature $T_{c}$, taken at the maximum of the DSC peak, with an estimated uncertainty of \pm $1^{\circ} \mathrm{C}$; (ii) the mass crystallinity ratio (or crystallinity) $X_{c}$, deduced from the area of the crystallization peak, with an absolute error about $\pm 1.5-2 \%$ for $X_{c} \sim 20-30 \%$. The theoretical enthalpy of crystallization was taken equal to $196 \mathrm{~J} / \mathrm{g}$ for the $\alpha$ phase of PA66. ${ }^{[22]}$ 

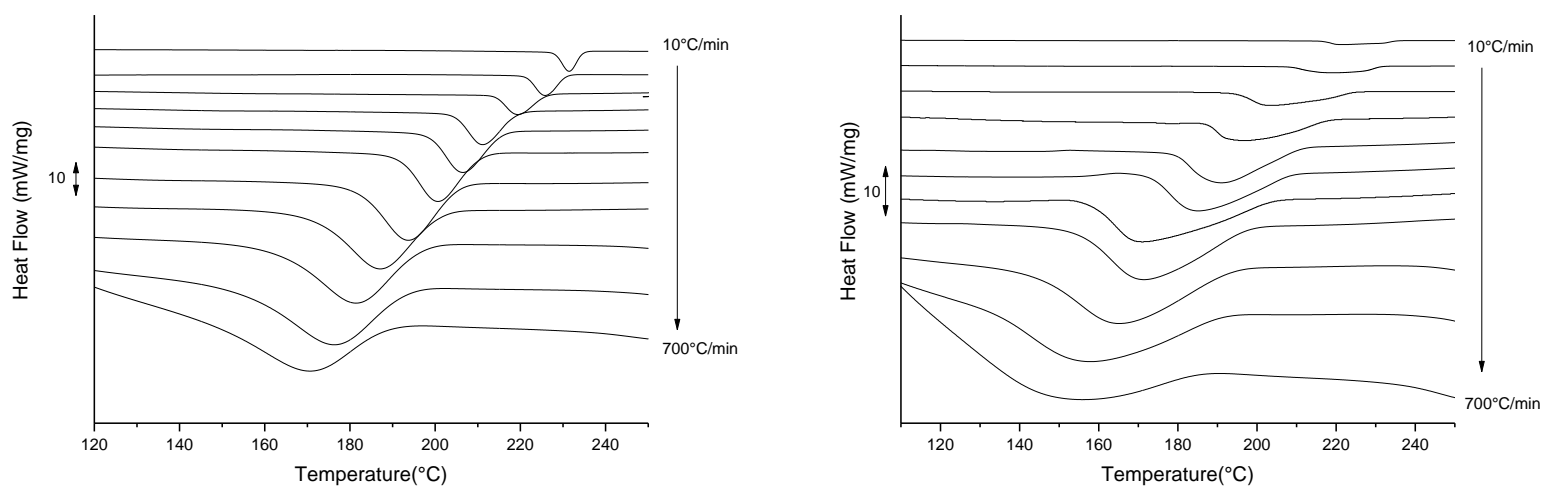

a

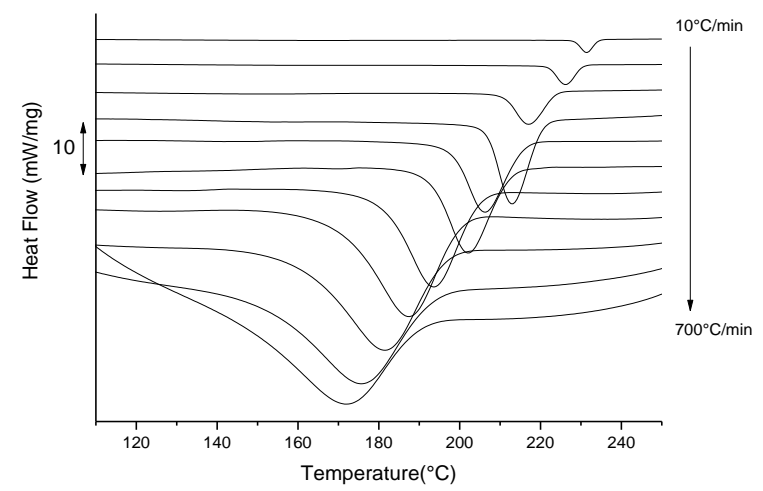

c

FIGURE 4 Crystallization thermograms: a) PA66-2; b) PA66-4; c) PA66-6. Cooling rates: $10,20,50,100,150,200,300,400,500,600,700^{\circ} \mathrm{C} / \mathrm{min}$

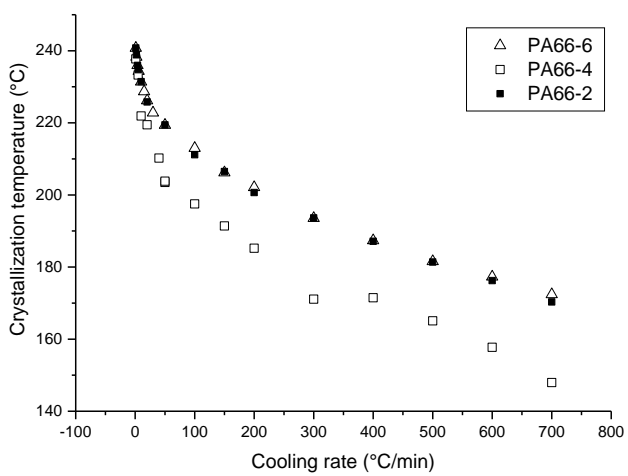

a

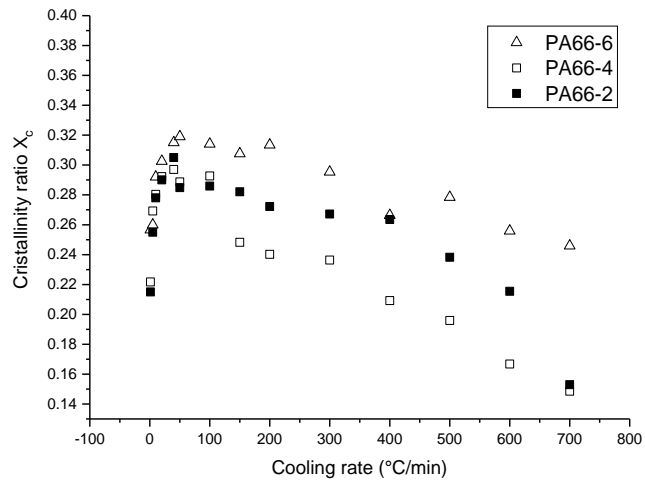

$\mathrm{b}$

FIGURE 5 Influence of cooling rate on crystallization temperature (a) and crystallinity ratio (b) 
For the three grades of PA66, crystallization temperature decreases with increasing cooling rate, as expected (Figure 5a). The crystallization temperatures of PA66-2 and PA66-6 are very close, whereas those of PA66-4 are lower. These lower temperatures can be related to the shoulder on the DSC curves, which induces a shift of the main peak to lower temperatures ${ }^{[18]}$ Crystallinity surprisingly increases when cooling rate increases from 1 to $50^{\circ} \mathrm{C} / \mathrm{min}$ and after a more or less pronounced plateau, decreases for further cooling-rate increase (Figure 5b). This initial increase, which is greater than the experimental error, is difficult to interpret: it suggests that there is a short cooling-rate range, corresponding to a crystallization-temperature range, for which nucleation and growth phenomena lead to a maximum crystallinity. Another explanation could be polymer degradation: during a slow cooling, the polymer remains longer at high temperature, which could induce some degradation, responsible for a decrease of crystallinity. Crystallinity of PA66-4 is significantly lower, which can be related to the higher molar mass of this polymer. This higher molar mass could also contribute to the lowering of crystallization temperatures. Conversely, branching does not seem to play any role. Again, the slight difference between the crystallinities of PA66-2 and PA66-6 could be attributed to differences in molar masses.

For each cooling rate $\dot{T}$, the transformed fraction at temperature $T, \alpha(T, \dot{T})$, is assimilated to the relative crystallinity:

$$
\alpha(T, \dot{T})=\frac{X(T)}{X\left(T_{\text {end }}\right)}=\frac{X(T)}{X_{c}}
$$

$X(T)$ represents the crystallinity developed between the onset of crystallization and temperature $T$, which is deduced from crystallization exotherms; $X\left(T_{\text {end }}\right)$ is the crystallinity at the end of crystallization, i.e., $X_{c}$ displayed in Figure 2b. The transformed fraction is plotted in Figure 6 for the three grades of PA66 and cooling rates of 10, 50, 150, 300, 700 ${ }^{\circ} \mathrm{C} / \mathrm{min}$. Crystallization overall kinetics of PA66-6 and PA66-2 are almost the same. PA66-4 crystallizes at lower temperatures and over a wider temperature range. 


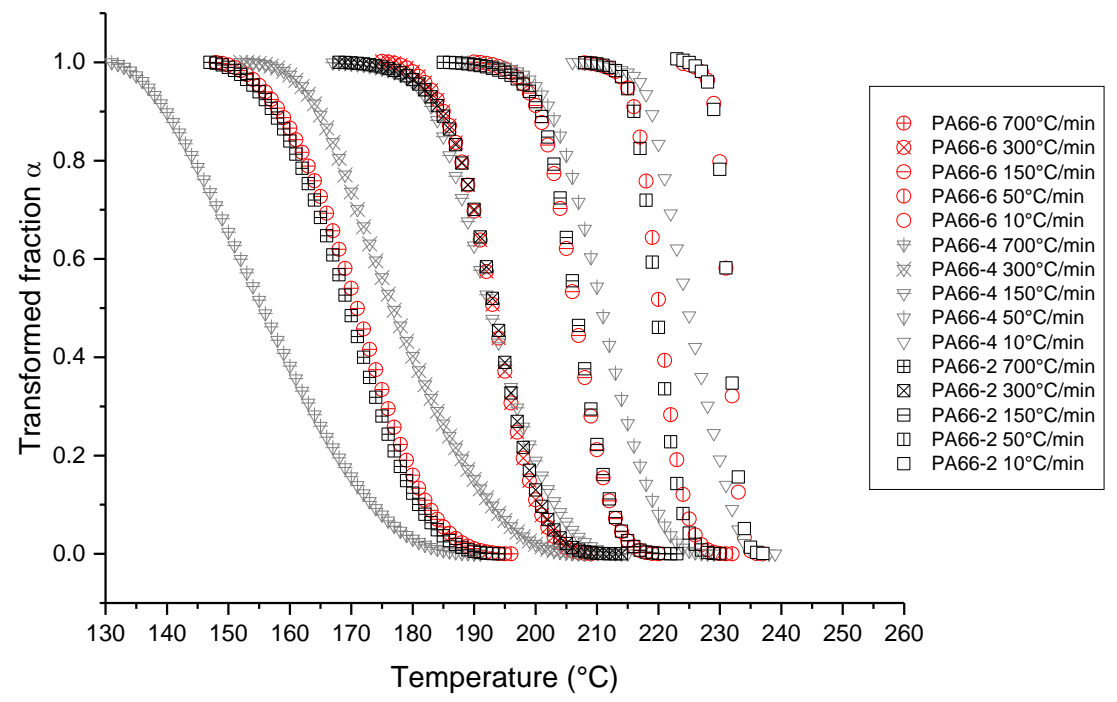

FIGURE 6 Transformed fractions for the three grades of PA66 and cooling rates of 10, 50, $150,300,700^{\circ} \mathrm{C} / \mathrm{min}$

$\alpha(T, \dot{T})$ is interpreted using Ozawa equation: ${ }^{[23]}$

$\alpha(T, \dot{T})=1-\exp \left[-\frac{\chi(T)}{\dot{T}^{n}}\right]$

with $n$ the Avrami exponent and $\chi(T)$ the Ozawa function. Equation 2 can be rewritten as:

$\ln [-\ln (1-\alpha(T, \dot{T}))]=\ln \chi(T)-n \ln \dot{T}$

Plotting $\ln [-\ln (1-\alpha(T, \dot{T}))]$ as a function of $\ln \dot{T}$ for various values of $T$ gives straight lines of slope $-n .^{[24]}$ Average values of $n$ are 3.9 and 3.7 for PA66-2 and PA66-6, respectively. These values, close to 4 , are consistent with a sporadic in time nucleation and a $3 \mathrm{D}$ spherulitic growth. ${ }^{[24]}$ Avrami exponent is 2.3 for PA66-4. This low value, associated to the existence of a shoulder on the crystallization curves, is attributed to transcrystallinity. Using these average values of $n \ln \chi(T)$ can be calculated for the three polymers and different $\dot{T}$ (Figure 7). Results for PA66-2 and PA66-6 are superimposed, while those for PA66-4 are quite different. Master curves can be drawn, described by polynomial expressions. For PA66-6: 
and for PA66-4:

$\ln \chi(T)=-2.23310^{-7} T^{4}+1.485310^{-4} T^{3}-3.742910^{-2} T^{2}+4.1454 T-162.10$

Finally, using Equation 2, we can calculate the overall crystallization kinetics for PA66-6 and PA66-4 and compare them to the experimental ones (Figure 8). The agreement is good.

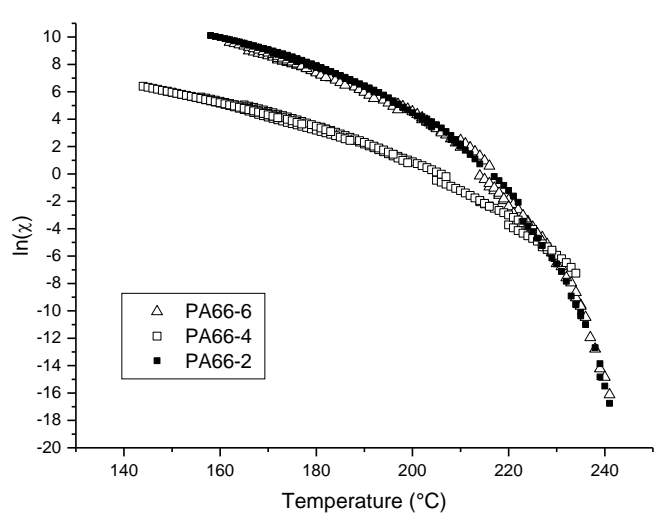

FIGURE $7 \ln \chi(T)$ curves
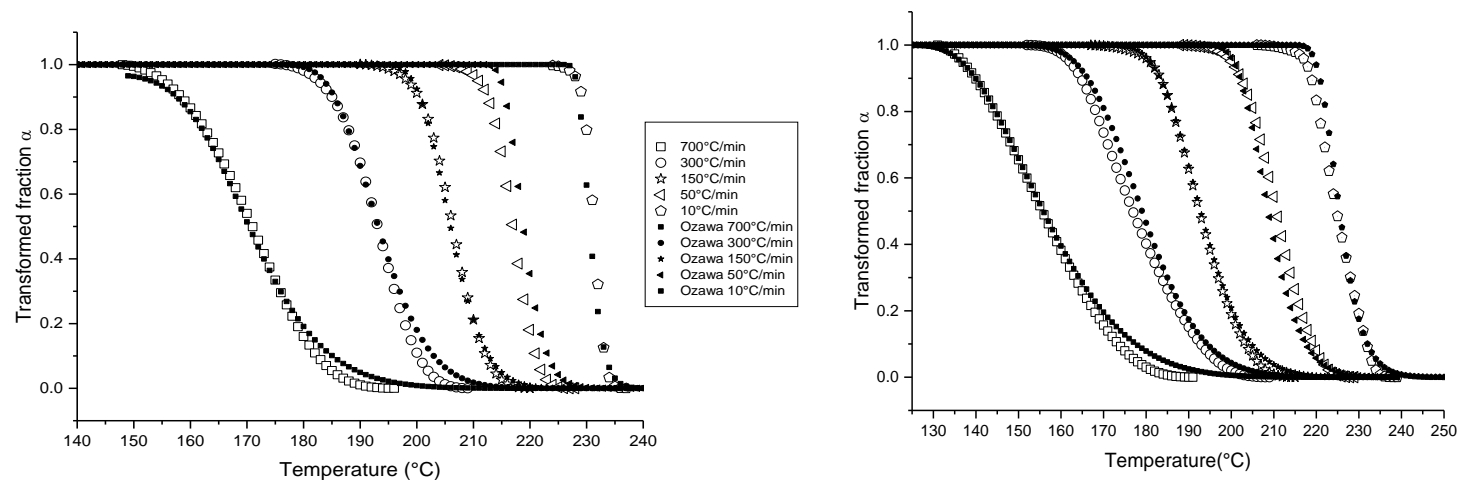

a

b

FIGURE 8 Comparison between measured (open symbols) and calculated (full symbols) crystallization kinetics: a) PA66-6; b) PA66-4 


\subsubsection{Optical microscopy}
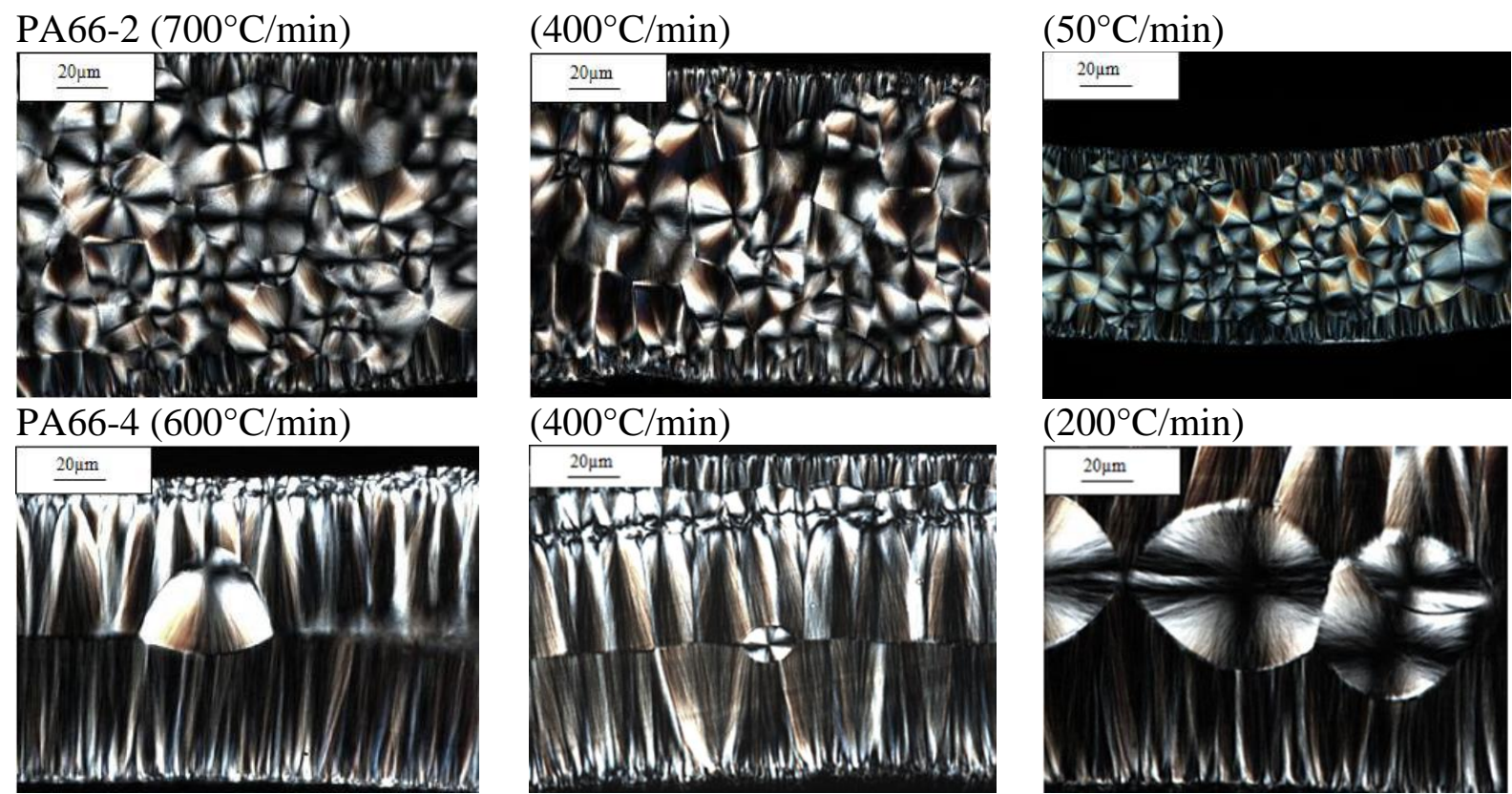

\section{PA66-6 $\left(700^{\circ} \mathrm{C} / \mathrm{min}\right)$}
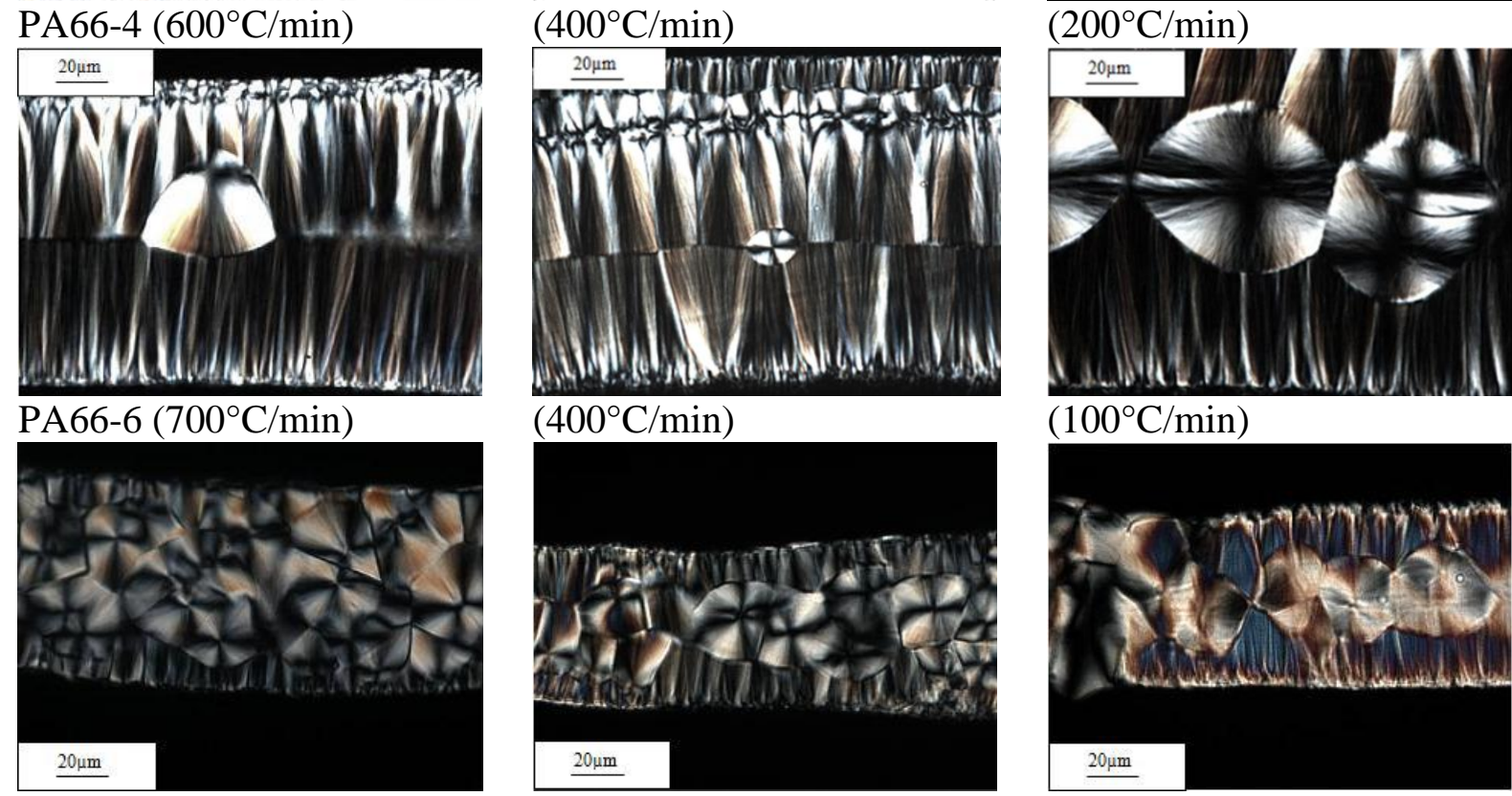

\section{$\left(400^{\circ} \mathrm{C} / \mathrm{min}\right)$}

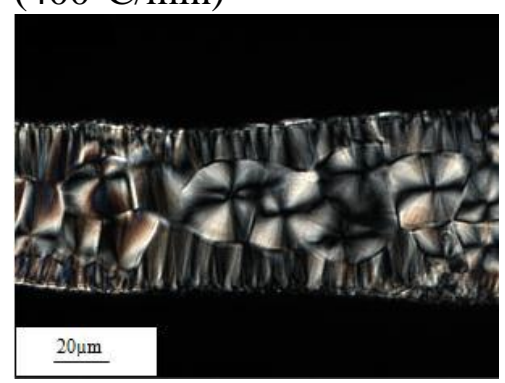

FIGURE 9 Microtomed cross sections of DSC specimens crystallized at different cooling rates

Observation of microtomed cross sections of PA66-2 and PA66-6 specimens shows similar morphologies (Fig. 9). Two transcrystalline zones start from the surfaces of polymer films, with a thickness of about $20 \mu \mathrm{m}$, due to the contact between the DSC aluminum pan and the polymer [18]. A more detailed comparison between PA66-2 and PA66-6 is difficult because the thicknesses of the films are not exactly the same, and as will be seen for PA66-4, an increase in the sample thickness increases core crystallization. Nevertheless, if we compare the size of spherulites in PA66-2 cooled at $50^{\circ} \mathrm{C} / \mathrm{min}$ and in PA66- 6 cooled at $100^{\circ} \mathrm{C} / \mathrm{min}$, we should have smaller spherulites in PA66-6 sample if crystallization in these two grades was exactly the same, which is not the case. Therefore, branching might have an effect on bulk nucleation, hence on transcrystallinity, but this should be confirmed by further investigation. This would be consistent with the slower spherulitic growth and volume crystallization that have been reported in the literature for branched polyamide [25]. Note that for treated glass 
slides, no transcrystalline zone was observed with these grades ( $\$ 3.1)$. This demonstrates a zone of polymer films, spherulitic morphologies are observed. The size of the spherulites decreases, while their number increases with increasing cooling rate. Therefore, overall kinetics is governed by spherulitic crystallization, which explains results obtained in 3.3.1.

Observation of microtomed cross sections of PA66-4 for the higher cooling rates (600 and $400^{\circ} \mathrm{C} / \mathrm{min}$ ) shows samples that are completely covered by transcrystallinity with a frontier between the two transcrystaline zones at the center of the polymer films, where we can also see one isolated spherulite. At lower cooling rate $\left(200^{\circ} \mathrm{C} / \mathrm{min}\right)$, more spherulites are observed between the two transcrystalline zones (Figure 9). The importance of transcristallinity in PA66-4 samples can explain the value of Avrami exponent close of 2 found above.

We can also notice in Figure 9 that for the sample of PA66-4 cooled at $200^{\circ} \mathrm{C} / \mathrm{min}$, one of the transcrystalline zones can be divided into two zones, one growing from the surface and the other starting at around $20 \mu \mathrm{m}$ from the surface. We can suppose that this two transcrystalline zones appear at almost the same time, the growth of surface transcristallinity being blocked by core transcrystallinity at $10 \mu \mathrm{m}$ from the surface. Then, core trancrystalline zone grows into the direction of the center, until it meets the opposite surface transcrystalline zone.

\subsection{3 | Synthesis}

Due to a high density of volume nuclei, PA66-2 and PA66-6 crystallize in the form of spherulites in the presence of surfaces of low nucleating activity ( $\$ 3.1$ ). In the case of active surfaces, e.g., surfaces of DSC pans, some transcrystallinity occurs, but it is blocked by the development of volume spherulites, as described previously. ${ }^{[20]}$

PA66-4 contains much less volume nuclei and is consequently more easily subjected to transcrystallinity in the presence of surfaces. This disturbs the recording of crystallization kinetics in DSC experiments, with the appearance of a shoulder on the crystallization curves. This sensivity to "accidental" nucleation could also explain the "double transcrystallinity" case in Figure 9. The counterpart is that this polymer is a good candidate for a detailed study of transcrystallinity. 


\subsection{Detailed analysis of transcrystallinity in PA66-4}

Instead of being only a drawback in the analysis of crystallization kinetics, transcrystallinity can be used to determine a number of crystallization parameters. From experimental work on HDPE films of different thicknesses, ${ }^{[20]}$ new methods have been proposed, which give access to crystallization parameters such as the number of nuclei per unit surface or the spherulite growth rate, and make it possible to determine the crystallization kinetics of the polymer not disturbed by transcrystallinity. Until now, these methods have been applied only to HDPE; ${ }^{[21]}$ they will be tested here on PA66-4.

\subsection{1 | Mathematical expressions}

Consider first transcrystallization between two parallel planes. At sufficiently high transformed volume fraction $\alpha$, there are two continuous fronts and $\alpha$ is given by:

$$
\alpha(t)=\frac{2}{e} \int_{0}^{t} G(u) d u
$$

Hence:

$$
\dot{T} e \frac{d \alpha}{d T}=2 G(T)
$$

where $T$ is the temperature, $\dot{T}$ the cooling rate and $e$ the sample thickness. As a consequence, experimental values of $0.5 \dot{T} e d \alpha / d T$ give access to the growth rate $G(T)$. The procedure is detailed in Reference [21].

Consider now a specimen containing both transcrystalline regions and spherulites at the core. The transformed volume $V_{\text {tran }}$ is decomposed into the volume $V_{\text {sur }}$ overlapped by transcrystallinity and the volume $V_{\text {vol }}$ occupied by bulk spherulites:

$$
V_{\text {tran }}=V_{\text {sur }}+V_{\text {vol }}=\alpha V_{\text {tot }}
$$


where $V_{t o t}$ is the total volume of the sample. The bulk crystallization can be characterized by $\alpha_{v}$, fraction of the volume which would be transformed if there were no transcrystallinity:

$\alpha_{v}=\frac{V_{v o l}}{V_{t o t}-V_{s u r}}$

which leads to:

$\alpha=\alpha_{v}+\left(1-\alpha_{v}\right) \frac{d}{e}$

where $d$ is the total thickness of the transcrystalline zones. So, when $\alpha e$ is plotted versus sample thickness at given temperature and cooling rate, it should obey a linear variation, with a slope $\alpha_{v}$.

\subsection{2 | Experimental results}

Experiments were performed with PA66-4 films of different thicknesses. All the samples had important transcrystalline zones at their surfaces. Figure 10 shows the crystallization curves obtained for three sample thicknesses: 162, 346 and $610 \mu \mathrm{m}$. The three DSC curves exhibit the shoulder associated with transcrystallinity, and it is possible to superimpose their hightemperature parts. In Figure 11 we can see that the thinnest sample is completely overlapped by transcrystallinity. The second sample contains one row of spherulites at the core, whereas more numerous bulk spherulites are observed in the third one.

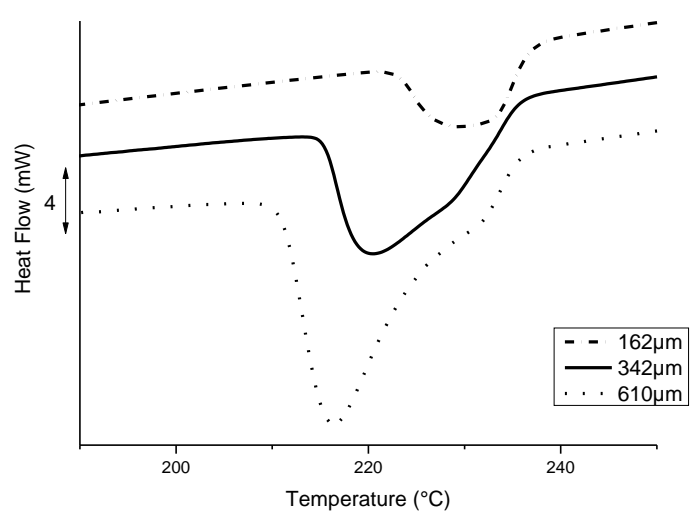

FIGURE 10 DSC crystallization curves of PA66-4 at a cooling rate of $10{ }^{\circ} \mathrm{C} / \mathrm{min}$ for different sample thicknesses: 162, 346 and $610 \mu \mathrm{m}$ 


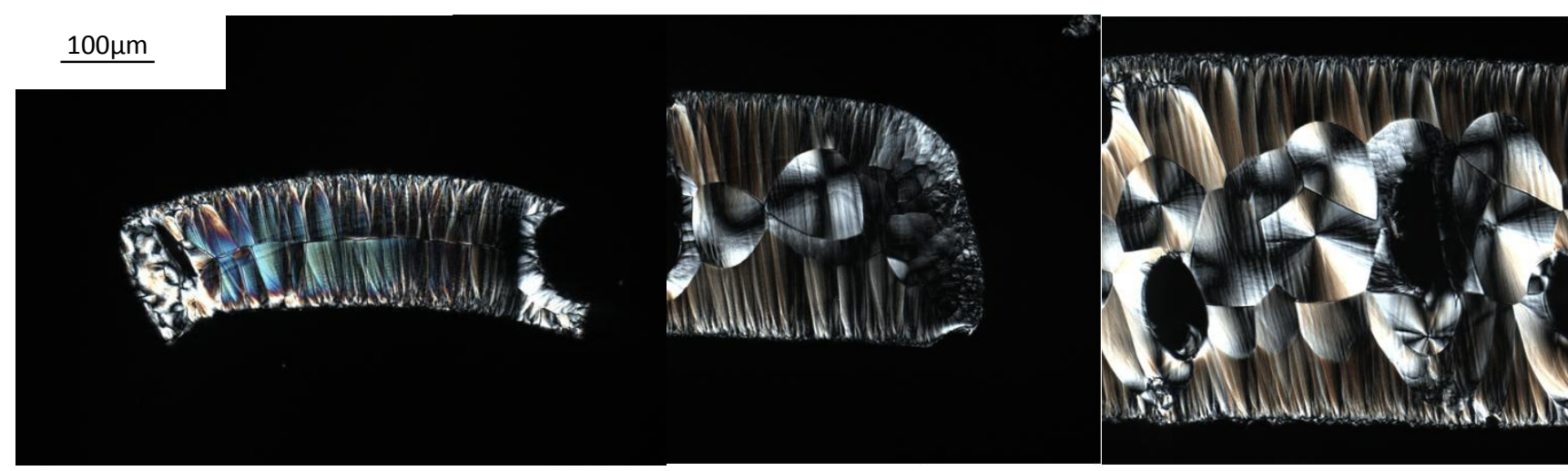

a

b

C

FIGURE 11 Microtomed cross sections of PA66-4 after DSC cooling at $10^{\circ} \mathrm{C} / \mathrm{min}$ for three different thicknesses: a) $162 \mu \mathrm{m}$; b) $346 \mu \mathrm{m}$; c) $610 \mu \mathrm{m}$

\subsection{3 | Growth rate data and interpretation}

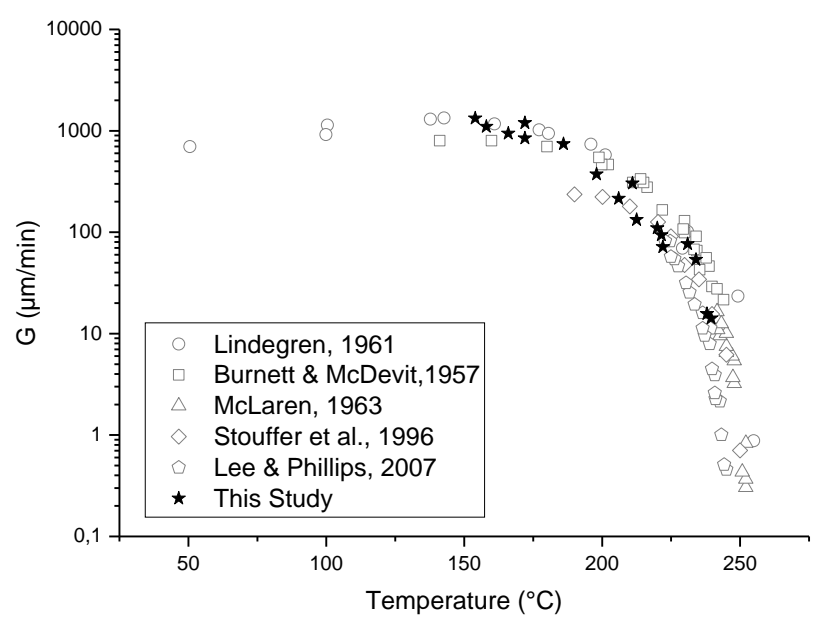

FIGURE 12 Growth rate of PA66 vs. temperature. Comparison between our results for PA66-4 (stars) and literature data

Growth rate has been determined as indicated above, using Equation 7. In Figure 12, our results (stars) are compared to literature data. There is a good agreement between our data and literature, which demonstrates the reliability of the method of determination.

Our experimental data can be interpreted using Hoffman-Lauritzen's equation: ${ }^{[16]}$ 
$\Delta T=T_{m}^{0}-T$ is the undercooling, with $T_{m}^{0}$ the equilibrium melting temperature, equal to $301^{\circ} \mathrm{C}^{[15]} U^{*}$ is the activation energy for the transport of molecular segments across the melt/crystal interface and $T_{\infty}$ is the temperature at which molecular mobility ceases. Usually, $U^{*}=6270 \mathrm{~J} \mathrm{~mol}^{-1}$ and $T_{\infty}=T_{g}-30, T_{g}$ being the glass transition temperature; here $T_{\infty}=$ $30^{\circ} \mathrm{C} . R$ is the gas constant, equal to $8.314 \mathrm{~J} \mathrm{~mol}^{-1} \mathrm{~K}^{-1} \cdot G_{0}^{i}$ and $K_{g}^{i}$ are constant for a given regime ( $\mathrm{i}=\mathrm{I}$, II, III).

Equation 11 can be rewritten as:

$\ln G+\frac{U^{*}}{R\left(T-T_{\infty}\right)}=\ln G_{0}^{i}-\frac{K_{g}^{i}}{T \Delta T}$

Therefore, for a given growth regime, the plot of $\ln G+\frac{U^{*}}{R\left(T-T_{\infty}\right)}$ as a function of $\frac{1}{T \Delta T}$ is a straight line, whose slope gives the value of $K_{g}^{i}$. Such a plot has been done for the results of Figure 12. It gives one straight line, which means one growth regime and one set of data: $K_{g}=$ $304075 \mathrm{~K}^{2}$ and $\ln G_{0}=14.02$.

Complementary results were obtained in high-temperature isothermal crystallizations, by directly measuring the increase of spherulite radii with time (Figure 13). Hoffman-Lauritzen's plot led to $K_{g}=504109 \mathrm{~K}^{2}$ and $\ln G_{0}=21.20$. As this value of $K_{g}$ is greater than that of the preceding paragraph, it is logically supposed that this high-temperature regime is regime I, whereas the low-temperature one is regime II (Figure 13). Furthermore, $K_{g}^{I} / K_{g}^{I I}=504109 / 304075 \sim 1.7$, which is close of the theoretical value of 2. From our data, we can estimate that we are in regime II below $239.5^{\circ} \mathrm{C}$ and in regime I above $245^{\circ} \mathrm{C}$. Similar results were obtained by Lee and Phillips. ${ }^{[15]}$ Nevertheless, they estimated that HoffmanLauritzen's theory was not appropriate to describe crystallization in positive spherulites, from the analysis of chain folding done by Lovinger. ${ }^{[9]}$ Lovinger found that in these spherulites, the 
$\boldsymbol{a}$-axis of the unit cell is parallel to the spherulite radius. The hydrogen bonding is in $(\boldsymbol{a}, \boldsymbol{c})$ planes in the direction of crystal growth $\boldsymbol{a}, \boldsymbol{c}$ being the chain axis. The chains are folding over in $(\boldsymbol{a}, \boldsymbol{c})$ planes along the radius of the spherulite, which causes their positive birefringence. In the description of crystallization given by Hoffman-Lauritzen's theory, chain folding should occur in plane parallel to the growth front, i.e., perpendicular to the radial growth direction. Further discussion of this point is without the scope of the present paper.

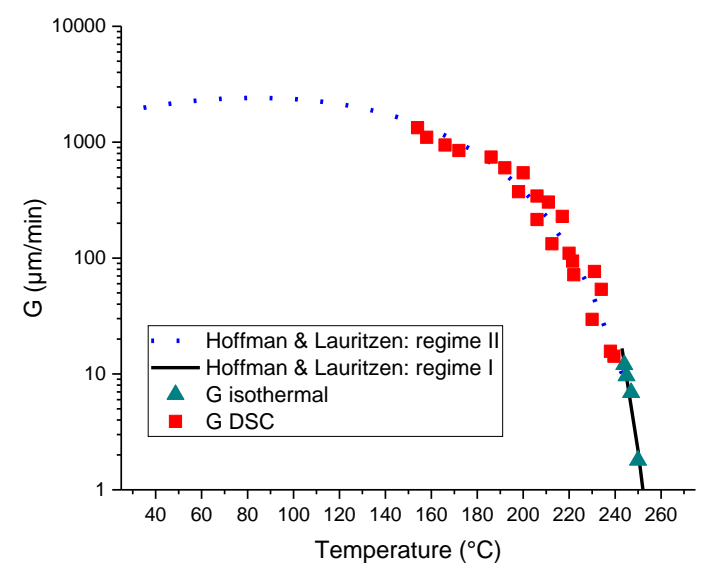

FIGURE 13 Growth rate of PA66-4 vs. temperature. Experimental data and interpretation in terms of Hoffman-Lauritzen's theory

\subsection{3 | Bulk crystallization kinetics}

The bulk transformed volume fraction $\alpha_{v}$ has been also successfully obtained (Figure 14). The plot of $\alpha e$ vs. sample thickness at given temperature and cooling rate actually gives a set of straight lines, whose slopes are the values of $\alpha_{v}$. Figure 15 makes it possible to appreciate the difference between the "true" overall kinetics $\alpha_{v}$ and the kinetics perturbed by more or less important transcrystallinity. 

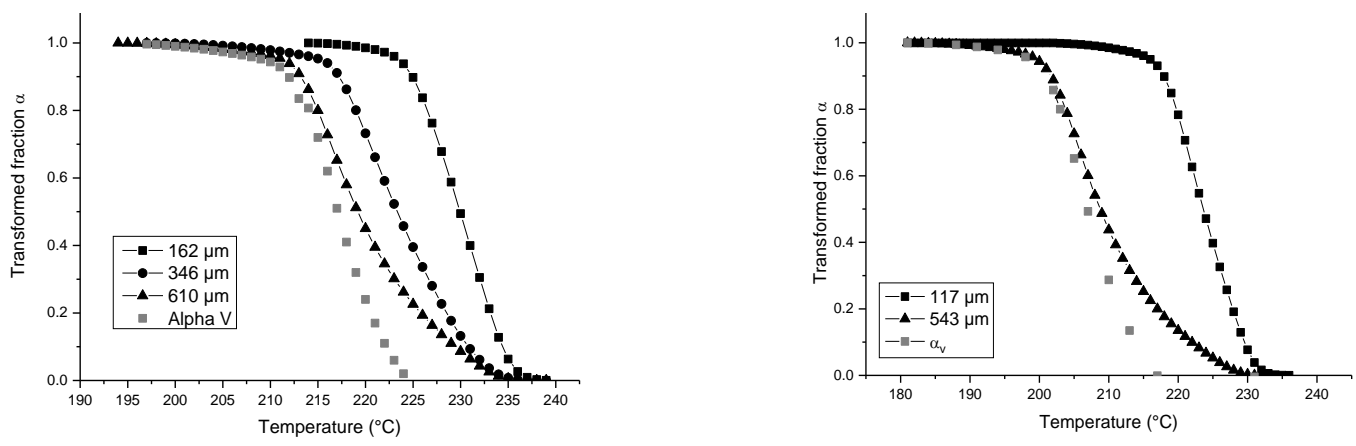

a

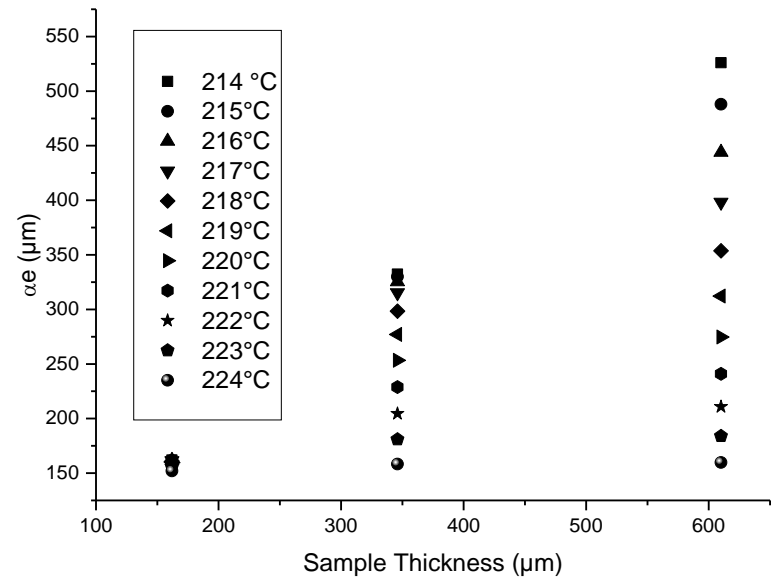

FIGURE 14 Determination of bulk crystallization at a cooling rate of $10^{\circ} \mathrm{C} / \mathrm{min}$ : $\alpha e$ vs. sample thickness for temperatures ranging from 214 to $224^{\circ} \mathrm{C}$
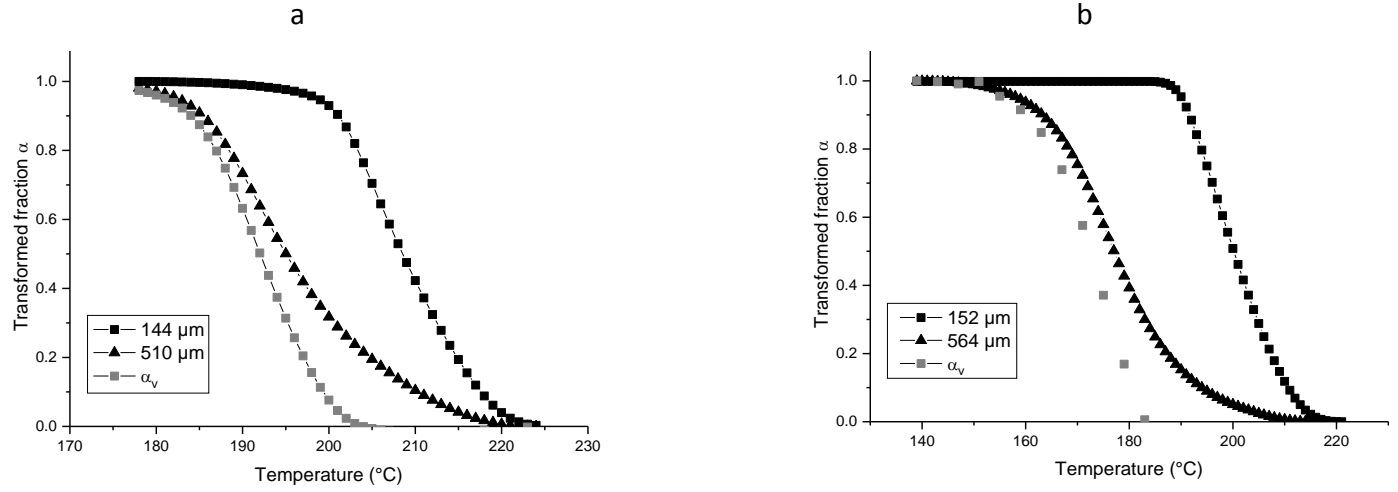

C

d

FIGURE 15 Evolution of bulk transformed volume fraction $\alpha_{v}$ vs. temperature at different cooling rates, and comparison with the kinetics for specimens of different thicknesses: a) $10^{\circ} \mathrm{C} / \mathrm{min}$; b) $20^{\circ} \mathrm{C} / \mathrm{min}$; c) $50^{\circ} \mathrm{C} / \mathrm{min}$; d) $100^{\circ} \mathrm{C} / \mathrm{min}$ 
Finally, for a cooling rate of $50^{\circ} \mathrm{C} / \mathrm{min}$, Figure 16 compares the overall crystallization $\alpha_{v}$. This figure allows a comparison of spherulitic crystallisation of the three polymers. That of PA66-4 is considerably slower. This has been attributed to the low number of volume nuclei (see Figure 3), probably due to a higher molar mass.

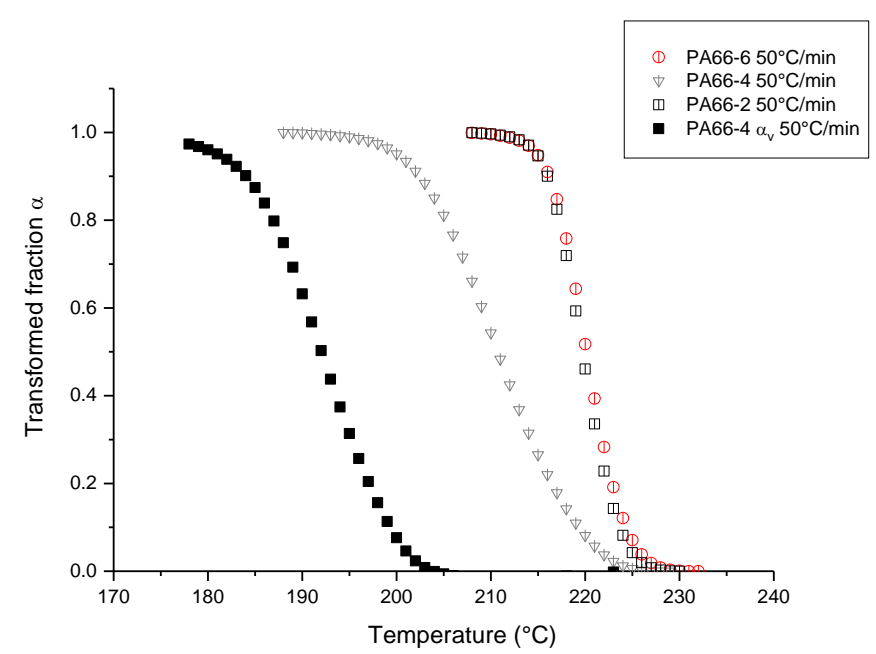

FIGURE 16 Comparison of the transformed fractions for the three grades of PA66 at a cooling rate of $50^{\circ} \mathrm{C} / \mathrm{min}$ and the bulk transformed volume fraction $\alpha_{v}$ for PA66-4 at the same cooling rate

\section{4 | CONCLUSION}

The crystallization behaviors of three experimental grades of polyamide 66 (PA66-2, PA66-4, PA66-6) have been compared. They mainly differ by their number average molar mass and their degree of branching. It appears that branching does not play any detectable role on crystallization temperatures and overall kinetics, a possible role on nucleation rate could be studied in further investigations. PA66-2 and PA66-6 are very close and quite different from PA66-4, which has the highest molar mass. Even if some transcrystallinity occurs in PA66-2 and PA66-6, their crystallization is mainly spherulitic. Conversely, PA66-4 is very sensitive to transcrystallinitity, which greatly disturbs its overall crystallization kinetics: a shoulder appears on the crystallization curves and the Avrami exponent is close to 2, instead of 4 for the two other spherulitic grades. This is attributed to a low number of bulk nuclei, in relation with high molar mass. As a global result, crystallinity of PA66-4 is lower, and its 
crystallization kinetics slower. These results underline the importance of controlling nucleation, e.g., through the use of nucleating agents.

DSC experiments can be used as model experiments to study transcrystallinity in thin polymer films. Varying the specimen thickness enables us to analyze the competition between surface and volume nucleation. Thus, in thin samples transcrystallinity is limited by the sample thickness. When thickness increases, the transcrystalline zones can grow, but up to a limiting value, because at a certain stage their development is stopped by the growth of bulk spherulites. The occurrence of transcrystallinity modifies the shape of crystallization curves, which exhibit a more or less pronounced shoulder. A change in the type of growth, from halfspheres to continuous fronts, is responsible for this shoulder observed in the DSC traces. All these statements have been experimentally verified in the present study.

A specific analysis of these DSC experiments gives access to crystallization parameters such as the growth rate of semi-crystalline entities. In spite of a certain degree of uncertainty, the results obtained by this new method compare well with already published data. This method is particularly of interest when direct observation of spherulites is impossible. Using the results given by this new method, as well as classical ones obtained by optical microscropy, made it possible to study the growth regimes in PA66-4.

It is also possible to determine the "intrinsic" crystallization kinetics of the polymer, i.e., not disturbed by transcrystallinity. This method has been applied to PA66-4. It quantitatively confirms the slow volume crystallization in this polymer. The kinetics obtained could be influenced by volume limitation, due to the small thickness of the specimens. This effect should not be too important here, since our experimental plots are in correct agreement with the corresponding theoretical expressions (linear plots). Nevertheless, theoretical developments should be necessary to appreciate the exact effect of confinement.

Indeed, another route to study both confinement and transcrystallinity effects is to model the overall kinetics in a thin film with and without transcrystallinity. Different models have been established in our laboratory ${ }^{[26-29]}$ and could be used for that purpose. These models are very important to understand the phenomena, but their effective application to a given 
polymer requires a number of data, not easy to determine, even if the present paper suggests original solutions.

\section{REFERENCES}

[1] C. W. Bunn, E. V. Garner, Proc. Roy. Soc. (London) 1947, 189A, 39.

[2] R. Brill, Z. phys. Chem. B 1943, 53, 61.

[3] C. Ramesh, A. Keller, S. J. E. A. Eltink, Polymer 1994, 35, 2483.

[4] M. L. Colclough, R. Baker, J. Mater. Sci. 1978, 13, 2531

[5] S. S. Lee, P. J. Phillips, Eur. Polym. J. 2007, 43, 1933.

[6] F. Khoury, J. Polym. Sci. 1958, 33, 389.

[7] J. H. Magill, J. Polym. Sci. Part A-2 1966, 4, 243.

[8] A. J. Lovinger, J. Appl. Phys. 1978, 49, 5003.

[9] A. J. Lovinger, J. Appl. Phys. 1978, 49, 5014.

[10] B. B. Burnett, W. F. McDevit, J. Appl. Phys. 1957, 28, 1101.

[11] C. R. Lindegren, J. Polym. Sci. 1961, 50, 181.

[12] J. V. McLaren, Polymer 1963, 4, 175.

[13] J. M. Stouffer, H. W. Starkweather Jr, B. S. Hsiao, P. Avakian, G. A. Jones, Polymer 1996, 37, 1217.

[14] Q. X. Zhang, Z. S. Mo, Chin. J. Polym. Sci. 2001, 19, 237.

[15] S. S. Lee, P. J. Phillips, Eur. Polym. J. 2007, 43, 1952.

[16] J. D. Hoffman, R. L. Miller, Polymer 1997, 38, 3151.

[17] C. Magnet, Cristallisation d'un polyamide 66 utilisé en filage textile. Influence d'un écoulement, $\mathrm{PhD}$ Thesis, Ecole Nationale Supérieure des Mines de Paris, France, 1993.

[18] N. Billon, C. Magnet, J. M. Haudin, D. Lefebvre, Colloid Polym. Sci. 1994, 272, 633.

[19] N. Billon, J. M. Haudin, J. Therm. Anal. 1994, 42, 679.

[20] N. Billon, V. Henaff, E. Pelous, J. M. Haudin, J. Appl. Polym. Sci. 2002, 86, 725.

[21] N. Billon, V. Henaff, J. M. Haudin, J. Appl. Polym. Sci. 2002, 86, 734.

[22] M. Inoue, J. Polym. Sci. Part A: General Papers 1963, 1, 2697.

[23] T. Ozawa, Polymer 1971, 12, 150.

[24] E. Piorkowska, A. Galeski, J. M. Haudin, Prog. Polym. Sci. 2006, 31, 549.

[25] S. Acierno, P.van Puyvelde, Polymer 2005, 46, 10331. 
[26] J. M. Escleine, B. Monasse, E. Wey, J. M. Haudin, Colloid Polym. Sci. 1984, 262, 366.

[27] N. Billon, J. M. Escleine, J. M. Haudin, Colloid Polym. Sci. 1989, 267, 668.

[28] N. Billon, J. M. Haudin, Colloid Polym. Sci. 1989, 267, 1064.

[29] A. Durin, J. L. Chenot, J. M. Haudin, N. Boyard, J. L. Bailleul, Europ. Polym. J. 2015, 73 December, 1.

\section{CAPTION FOR THE TABLE}

TABLE 1 Grades of PA66 studied.

\section{CAPTIONS FOR THE FIGURES}

FIGURE 1 Observation by optical microscopy of the morphologies in PA66-4 after isothermal crystallization at $244^{\circ} \mathrm{C}$.

FIGURE 2 Observation of the cross sections of films after isothermal crystallization at 244 C: a) PA66-2; b) PA66-4; c) PA66-6. Scale bar: $100 \mu \mathrm{m}$

FIGURE 3 Observation of the cross sections of pellets after crystallization during a slow cooling: a) PA66-2; b) PA66-4; c) PA66-6. Scale bar: $200 \mu \mathrm{m}$

FIGURE 4 Crystallization thermograms: a) PA66-2; b) PA66-4; c) PA66-6. Cooling rates: $10,20,50,100,150,200,300,400,500,600,700^{\circ} \mathrm{C} / \mathrm{min}$

FIGURE 5 Influence of cooling rate on crystallization temperature (a) and crystallinity ratio (b)

FIGURE 6 Transformed fractions for the three grades of PA66 and cooling rates of 10, 50, $150,300,700^{\circ} \mathrm{C} / \mathrm{min}$

FIGURE $7 \ln \chi(T)$ curves

FIGURE 8 Comparison between measured (open symbols) and calculated (full symbols) crystallization kinetics: a) PA66-6; b) PA66-4

FIGURE 9 Microtomed cross sections of DSC specimens crystallized at different cooling rates

FIGURE 10 DSC crystallization curves of PA66-4 at a cooling rate of $10{ }^{\circ} \mathrm{C} / \mathrm{min}$ for different sample thicknesses: 162, 346 and $610 \mu \mathrm{m}$ 
FIGURE 11 Microtomed cross sections of PA66-4 after DSC cooling at $10^{\circ} \mathrm{C} / \mathrm{min}$ for three different thicknesses: a) $162 \mu \mathrm{m}$; b) $346 \mu \mathrm{m}$; c) $610 \mu \mathrm{m}$

FIGURE 12 Growth rate of PA66 vs. temperature. Comparison between our results for PA66-4 (stars) and literature data

FIGURE 13 Growth rate of PA66-4 vs. temperature. Experimental data and interpretation in terms of Hoffman-Lauritzen's theory

FIGURE 14 Determination of bulk crystallization at a cooling rate of $10^{\circ} \mathrm{C} / \mathrm{min}$ : $\alpha e$ vs. sample thickness for temperatures ranging from 214 to $224^{\circ} \mathrm{C}$

FIGURE 15 Evolution of bulk transformed volume fraction $\alpha_{v}$ vs. temperature at different cooling rates, and comparison with the kinetics for specimens of different thicknesses: a) $10^{\circ} \mathrm{C} / \mathrm{min}$; b) $20^{\circ} \mathrm{C} / \mathrm{min}$; c) $50^{\circ} \mathrm{C} / \mathrm{min}$; d) $100^{\circ} \mathrm{C} / \mathrm{min}$

FIGURE 16 Comparison of the transformed fractions for the three grades of PA66 at a cooling rate of $50^{\circ} \mathrm{C} / \mathrm{min}$ and the bulk transformed volume fraction $\alpha_{v}$ for PA66-4 at the same cooling rate 


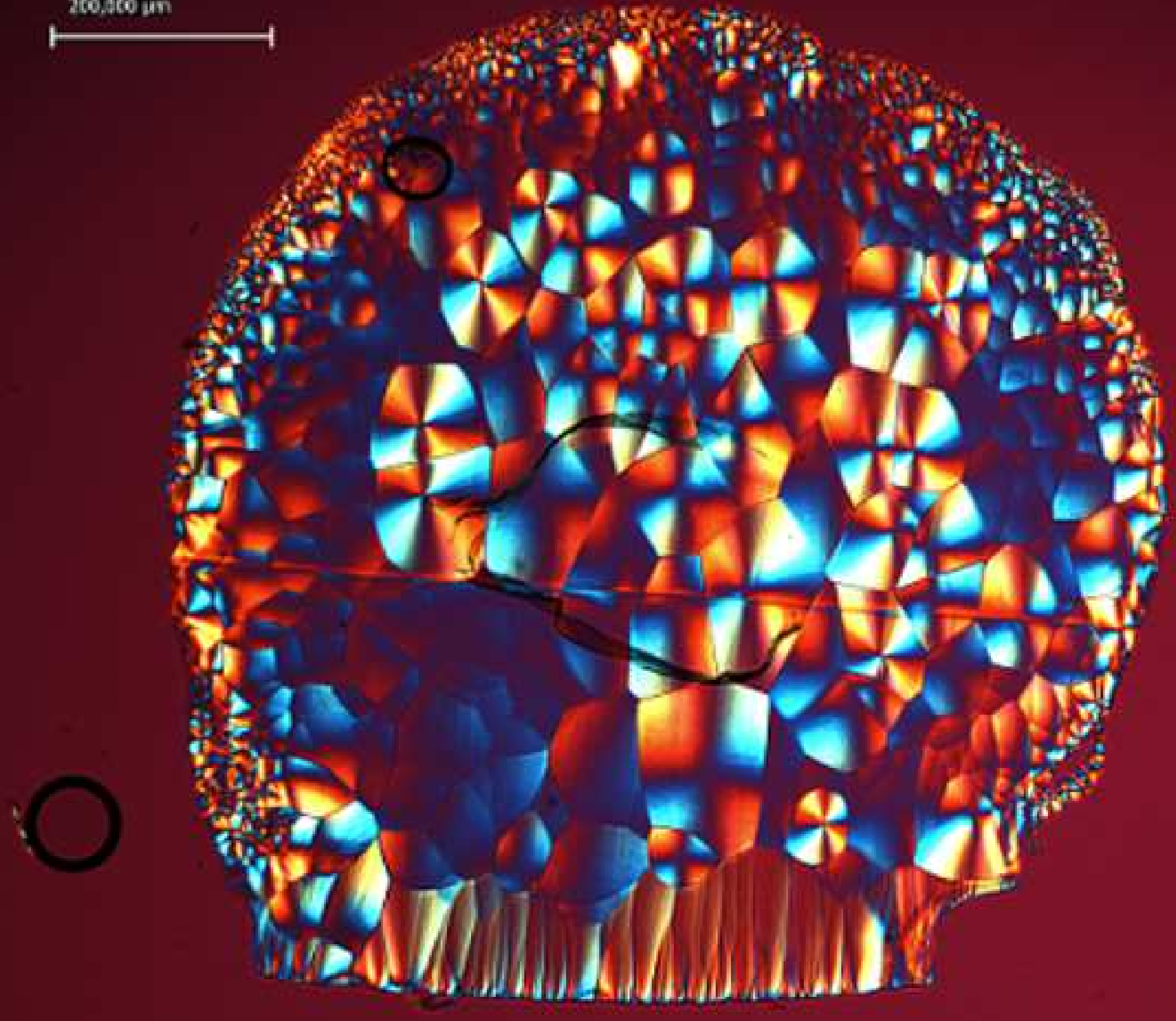




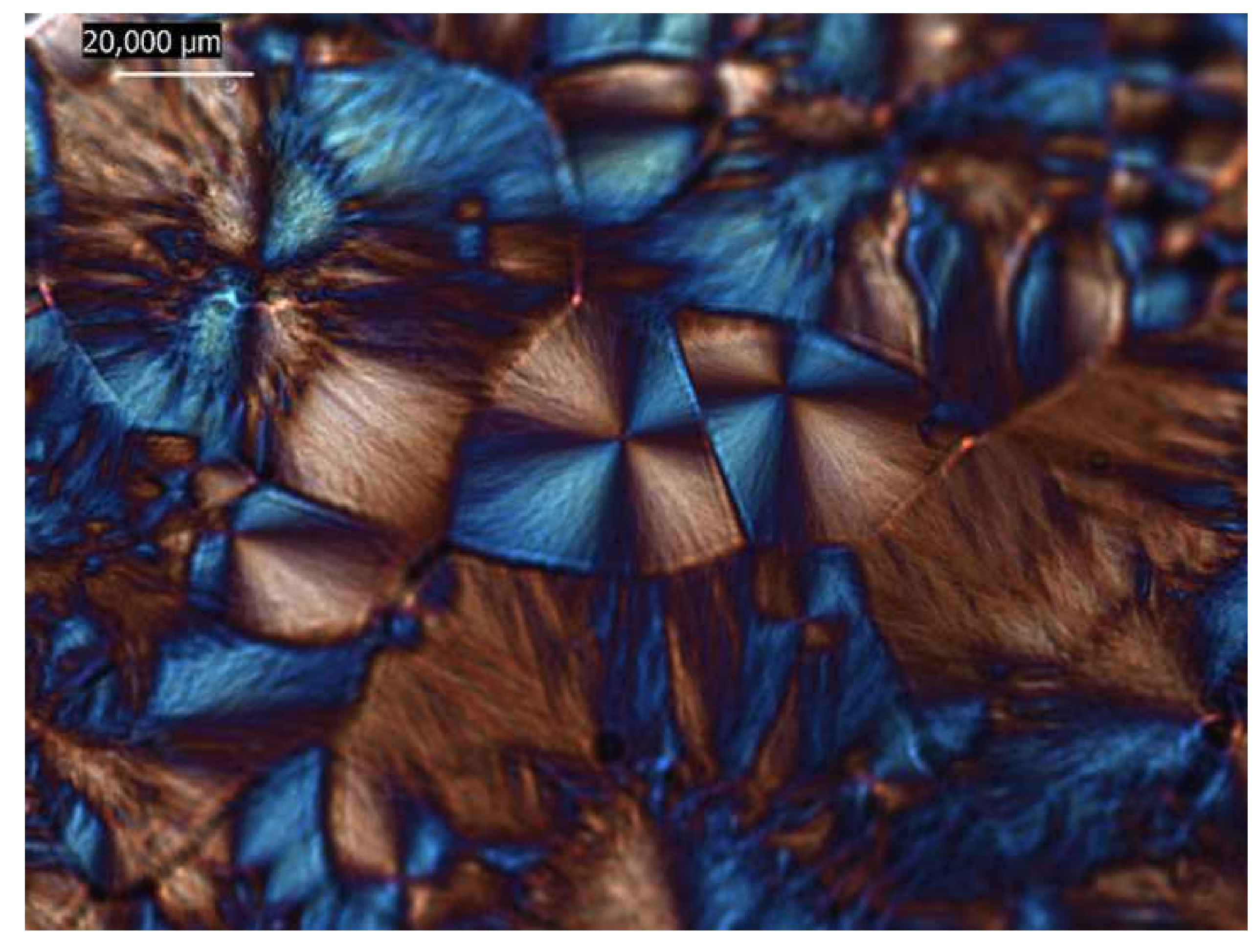

\section{$\underline{\Delta}$}
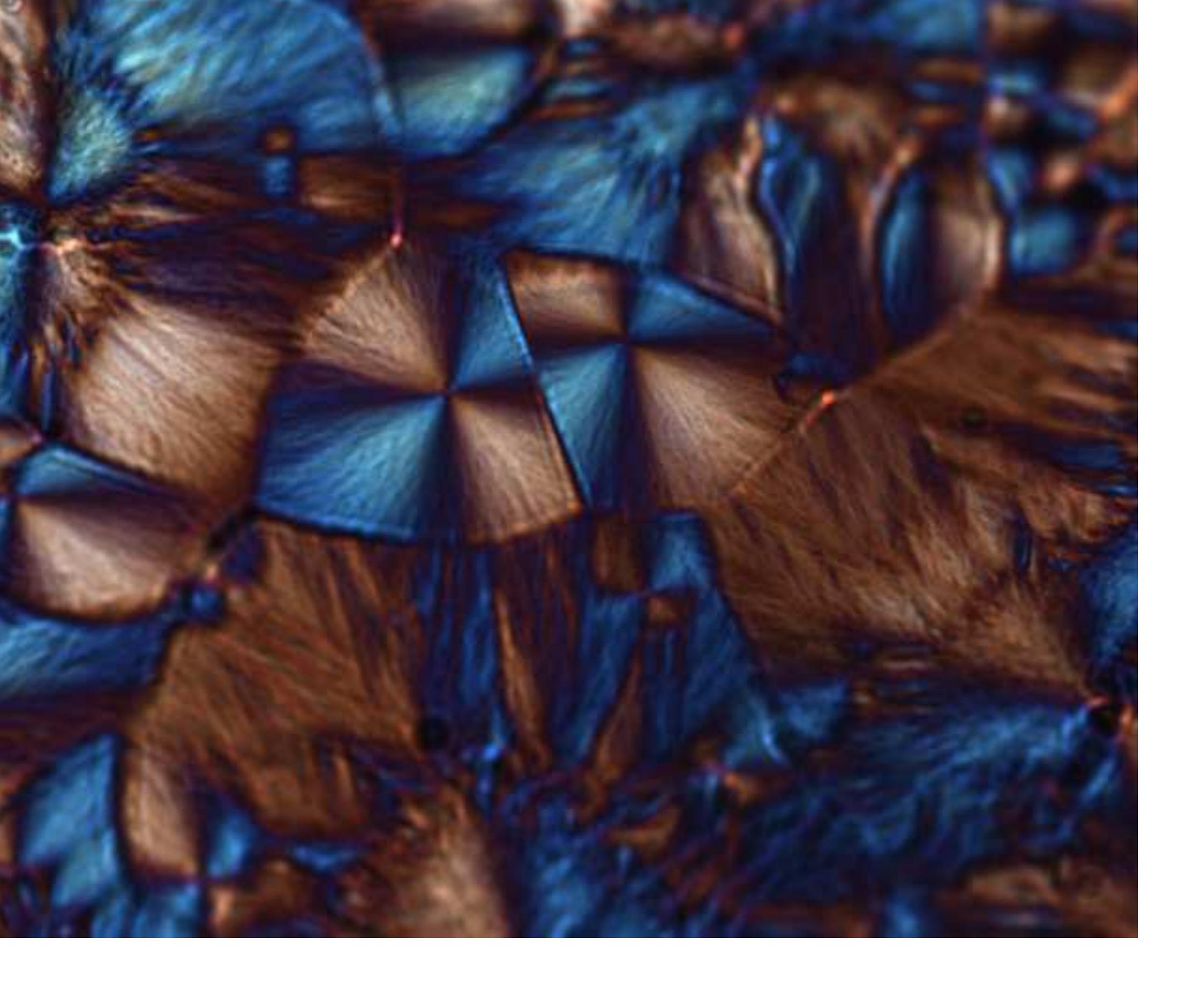


\section{$100,0 \mu \mathrm{m}$}

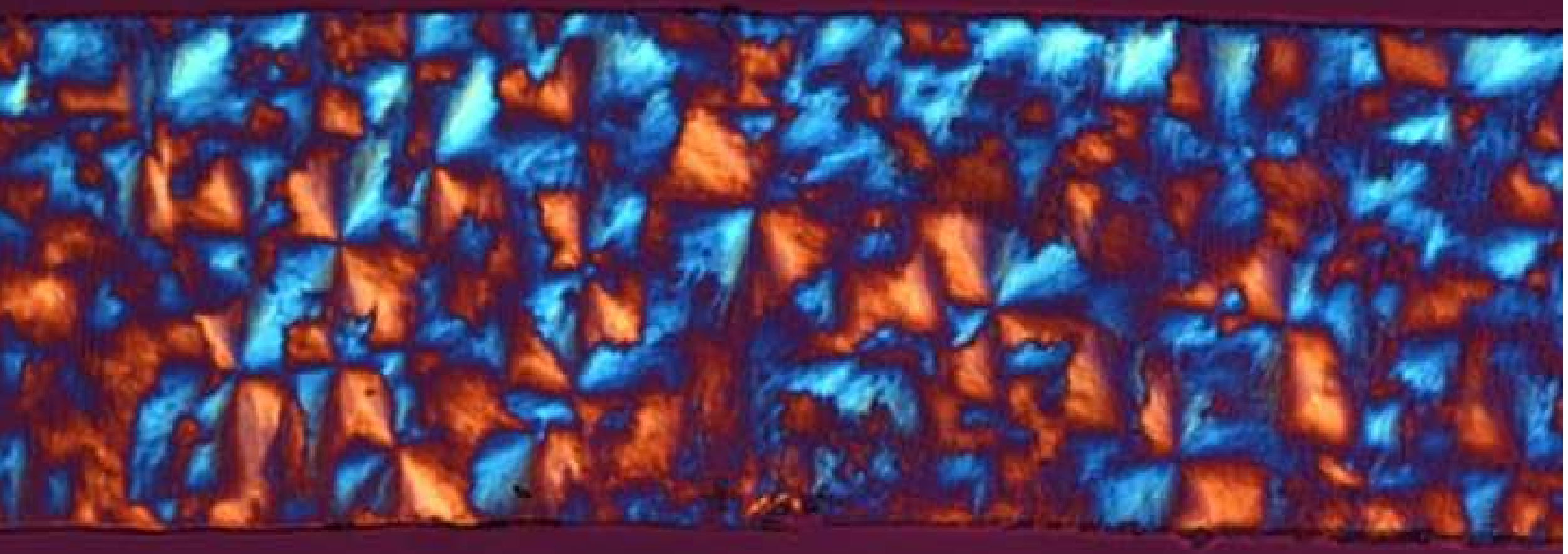




\section{$100,0 \mu \mathrm{m}$ \\ $\longmapsto$}

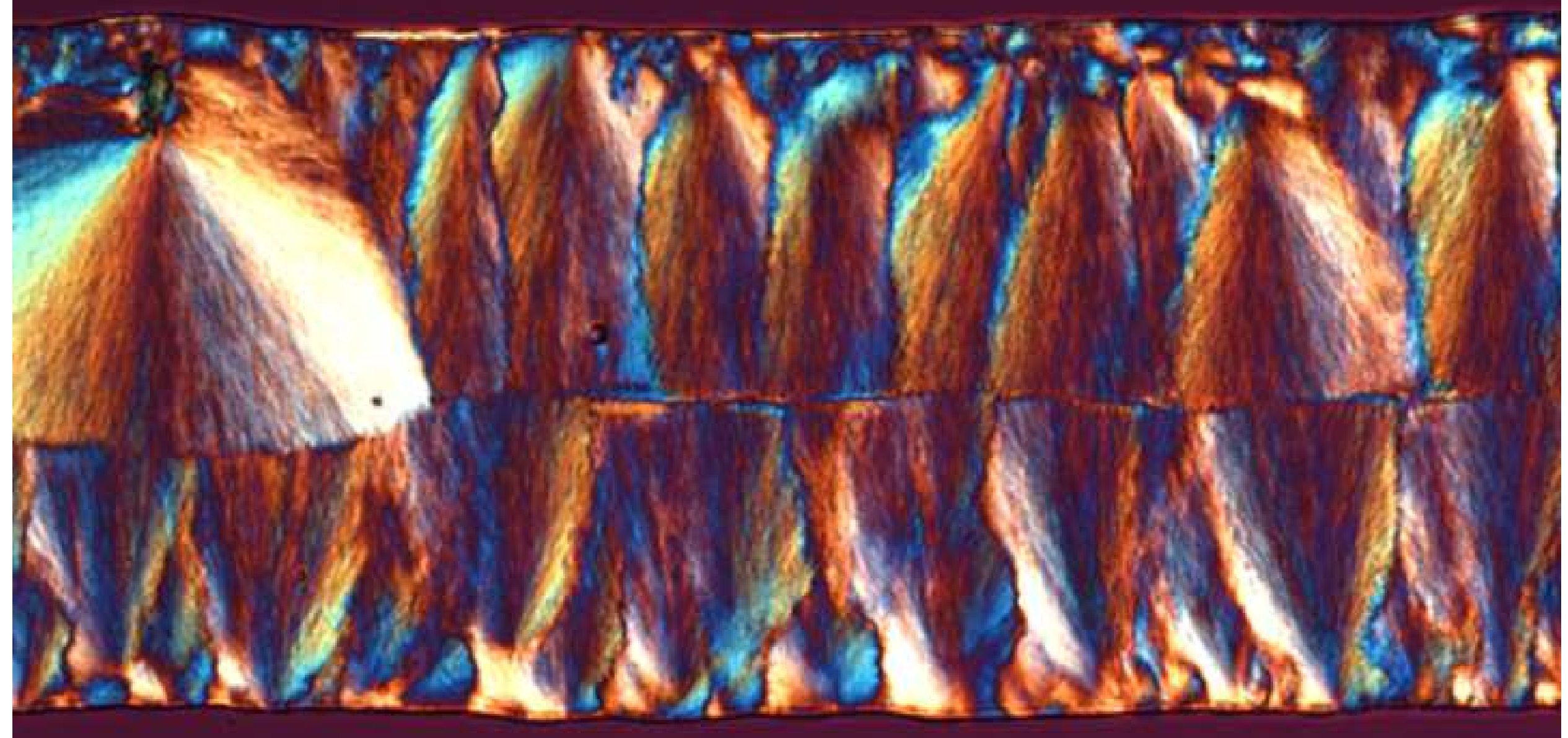




$\longmapsto$
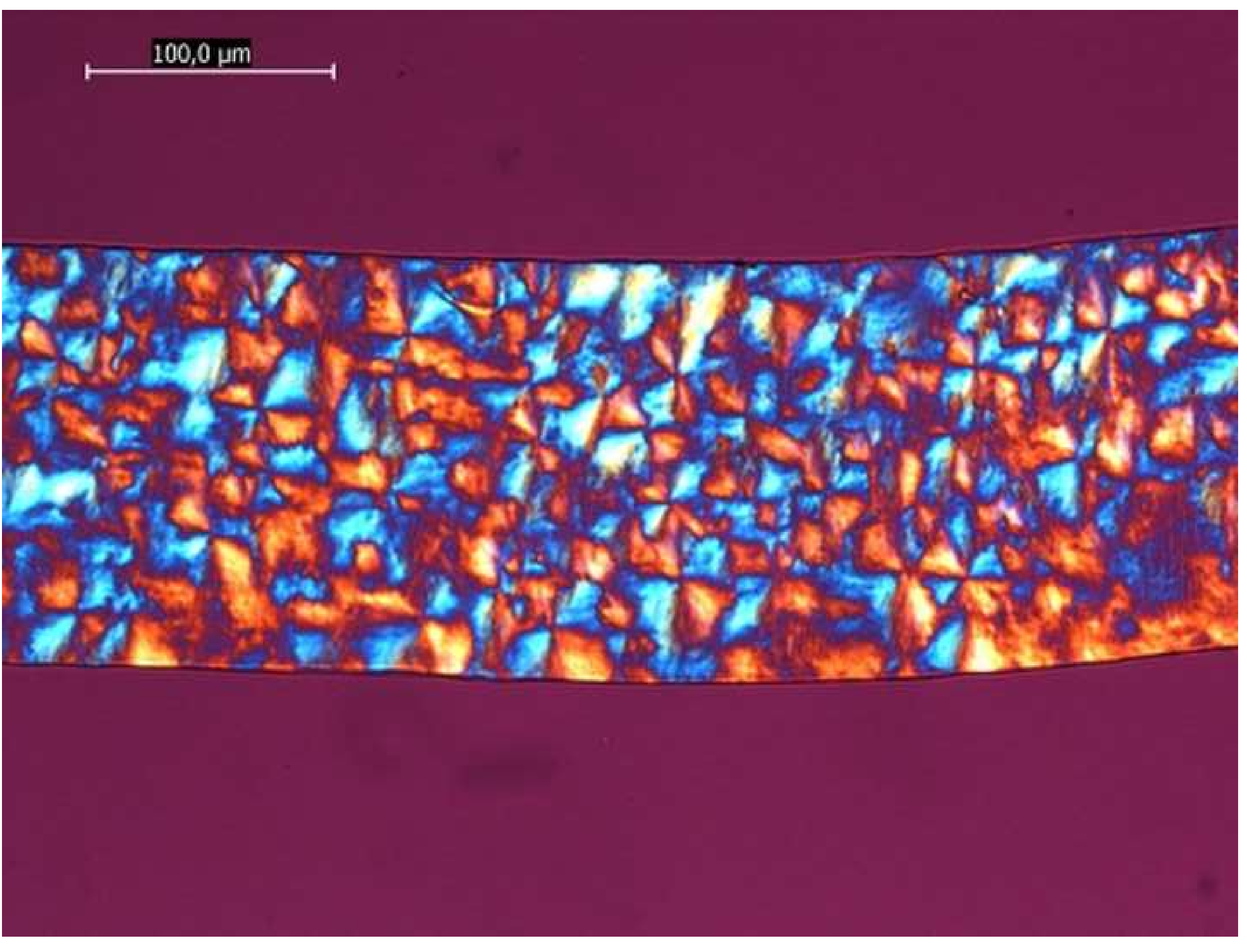


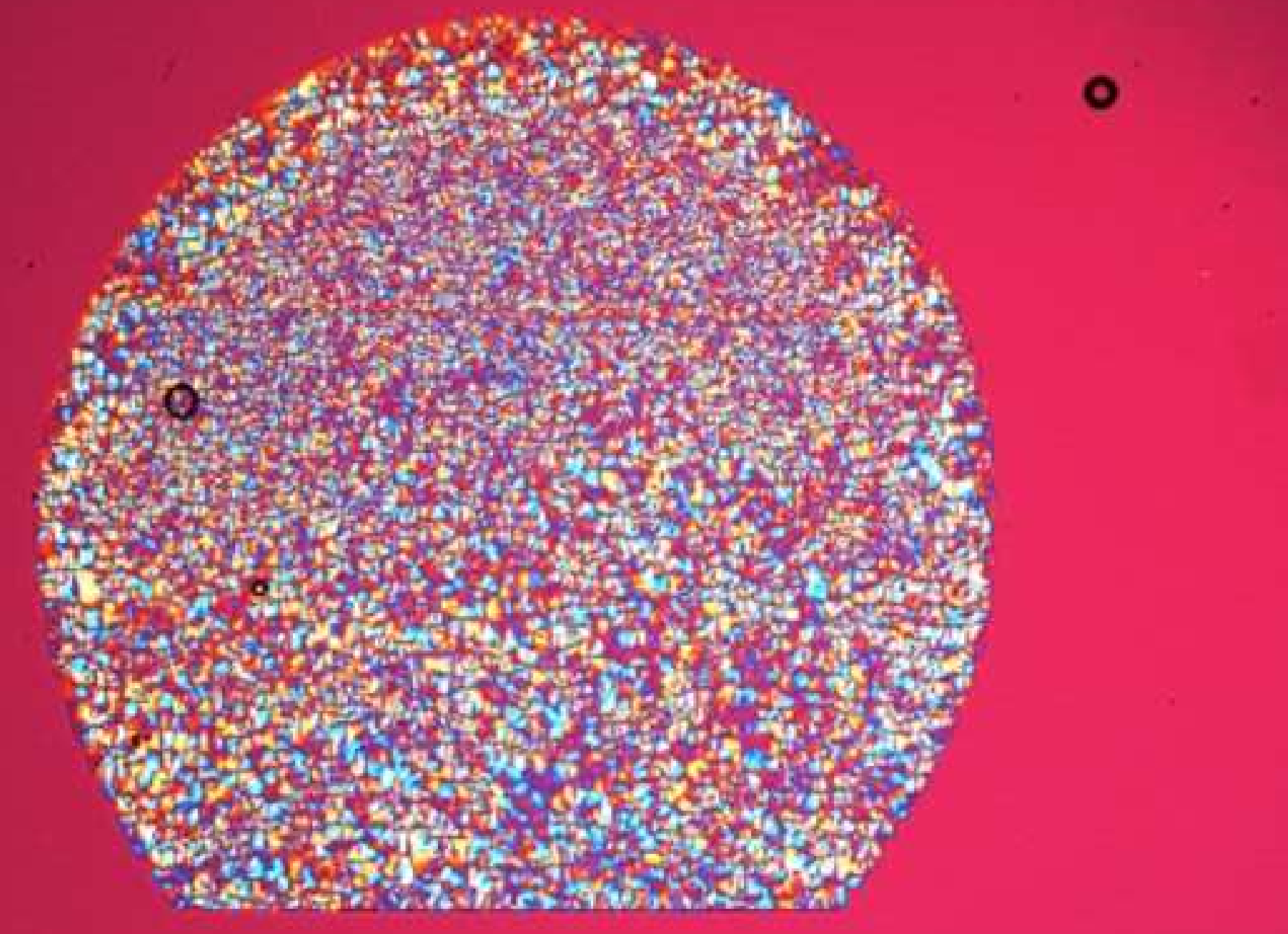

○ 


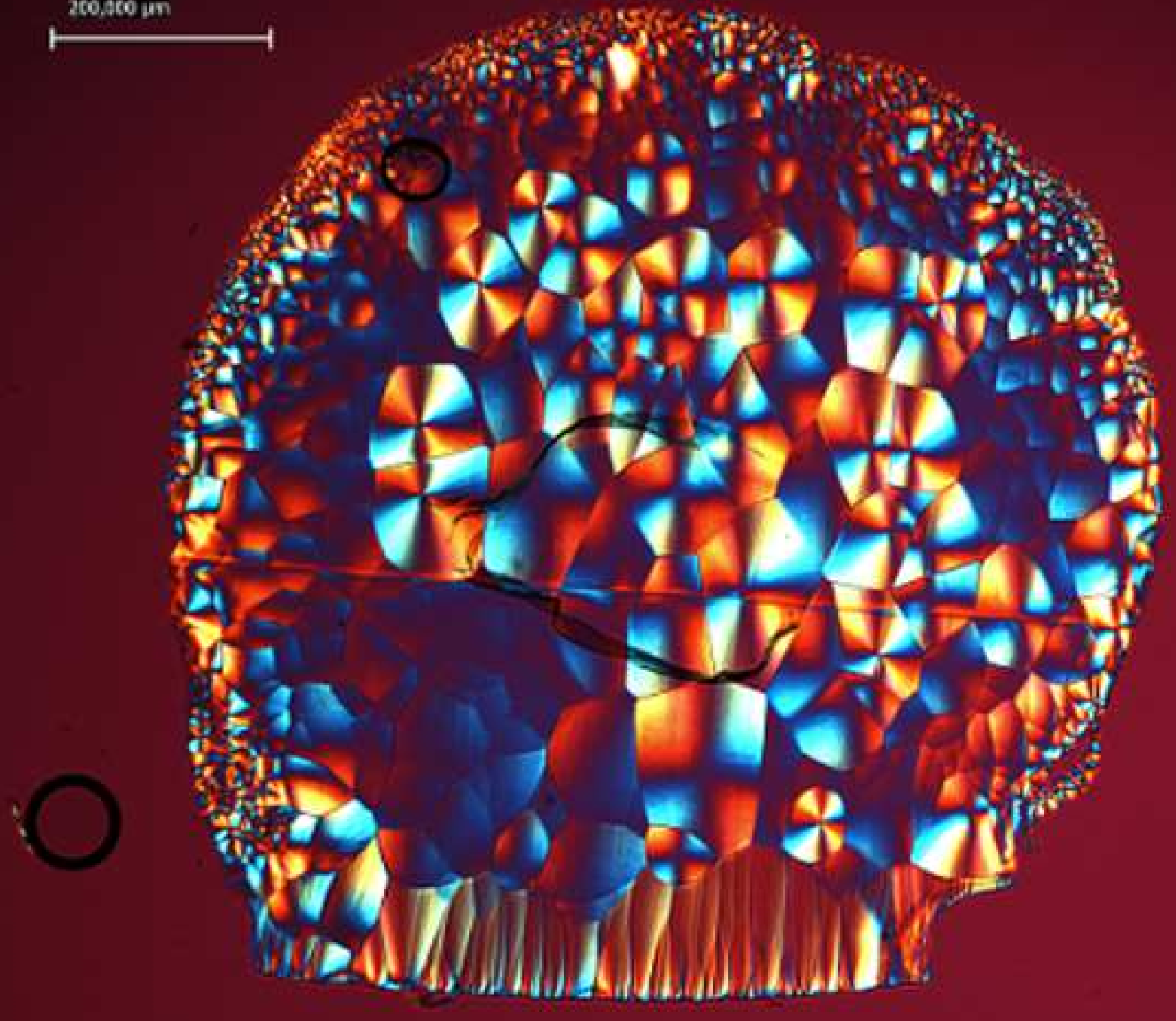




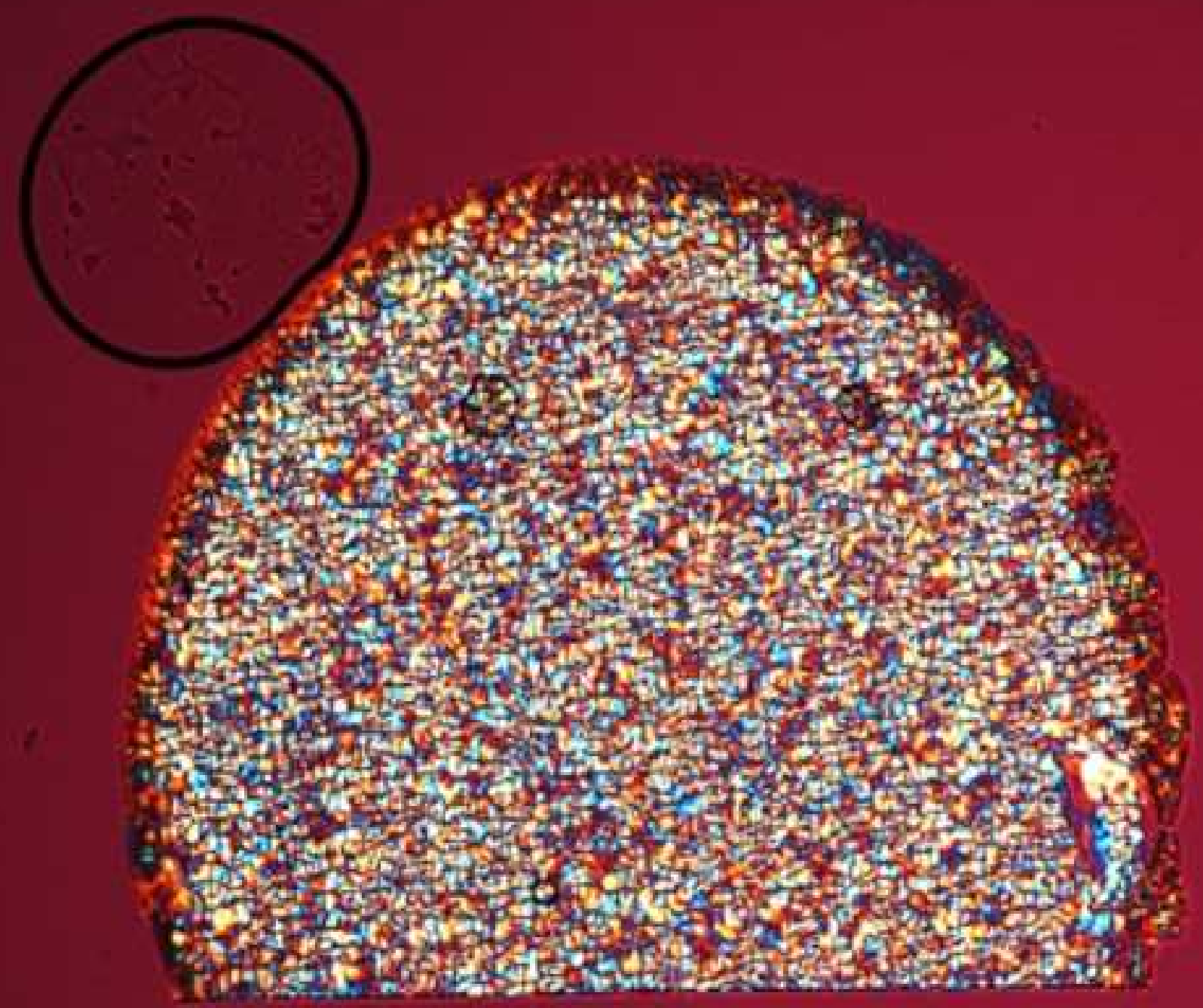




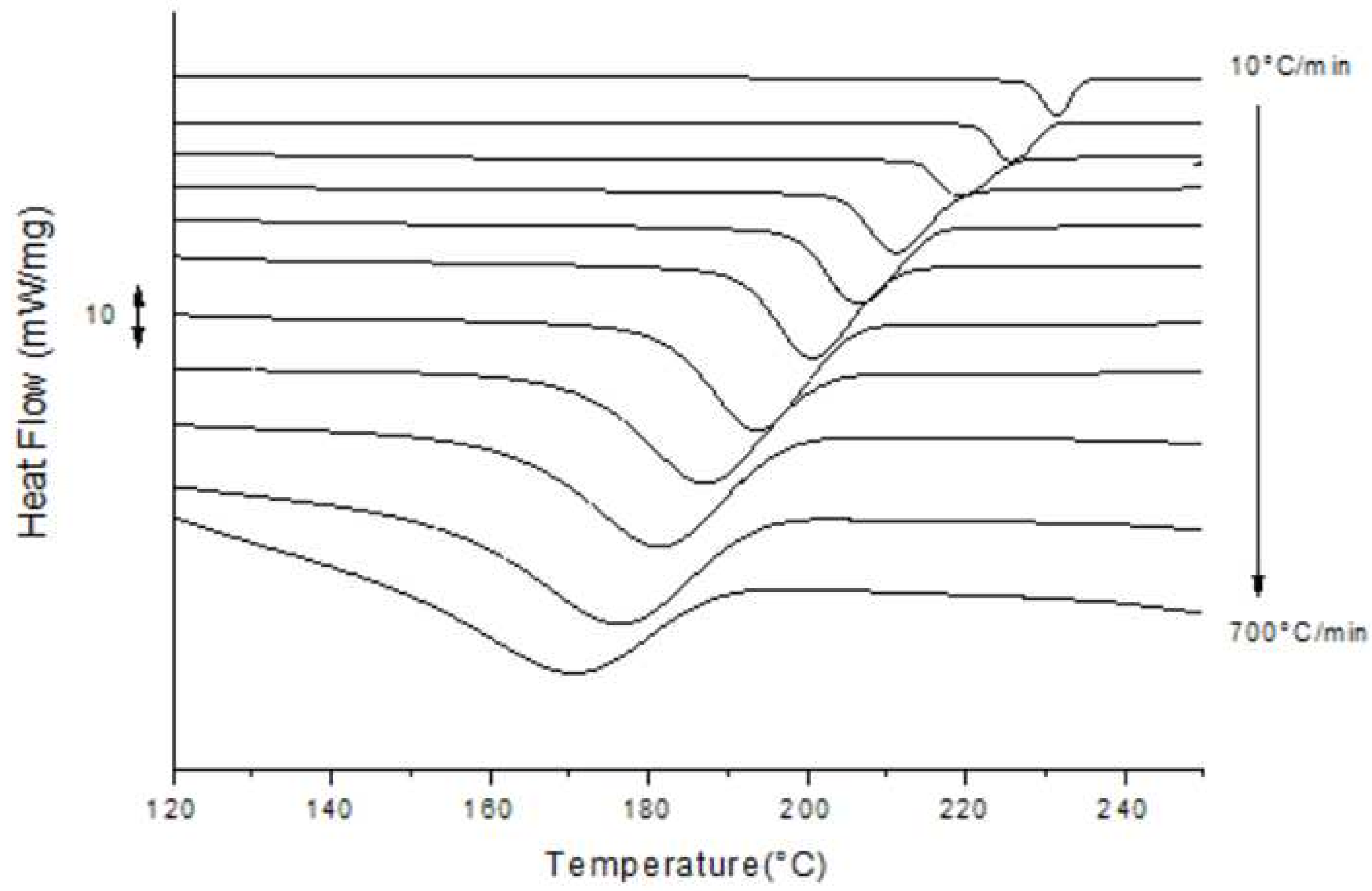




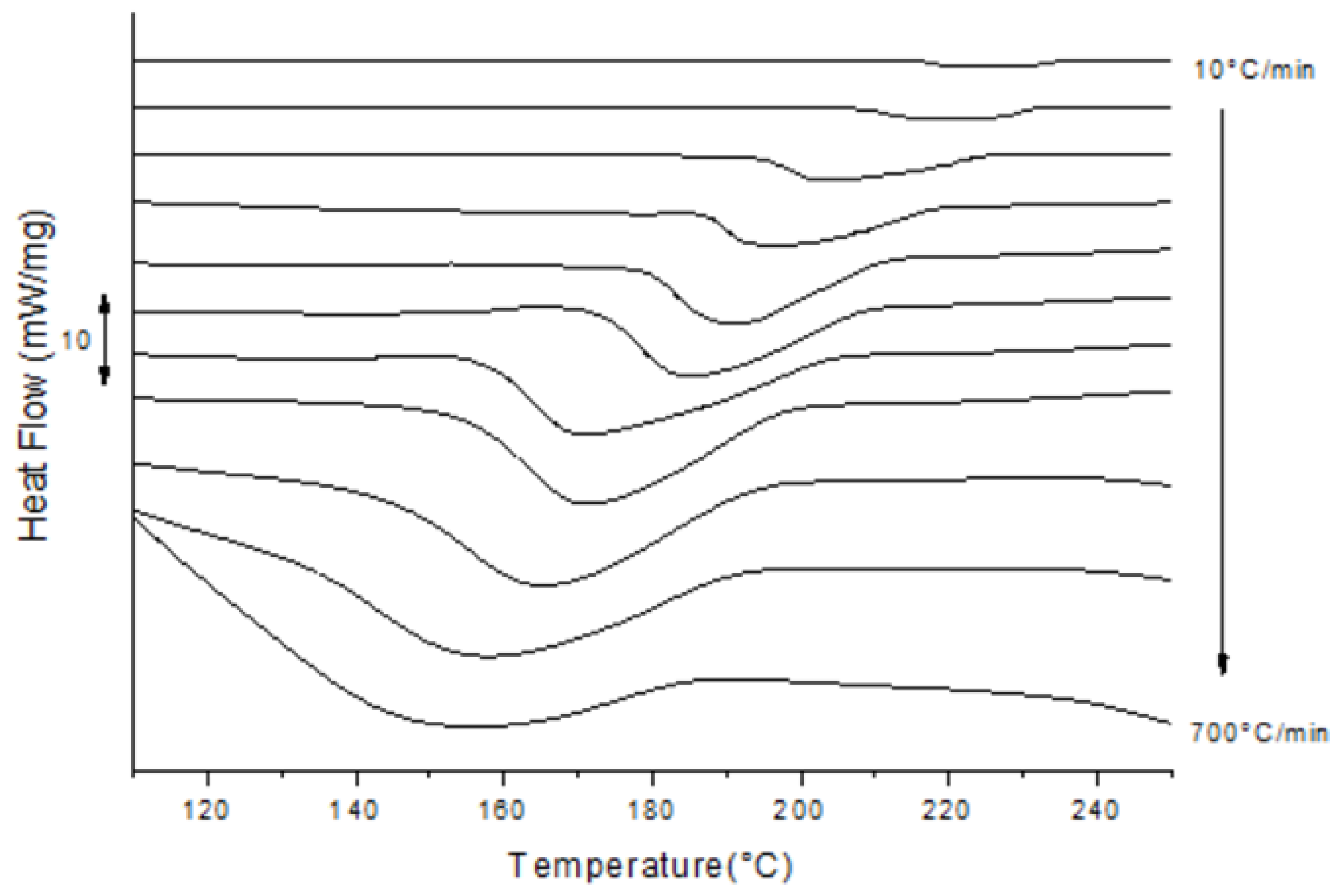




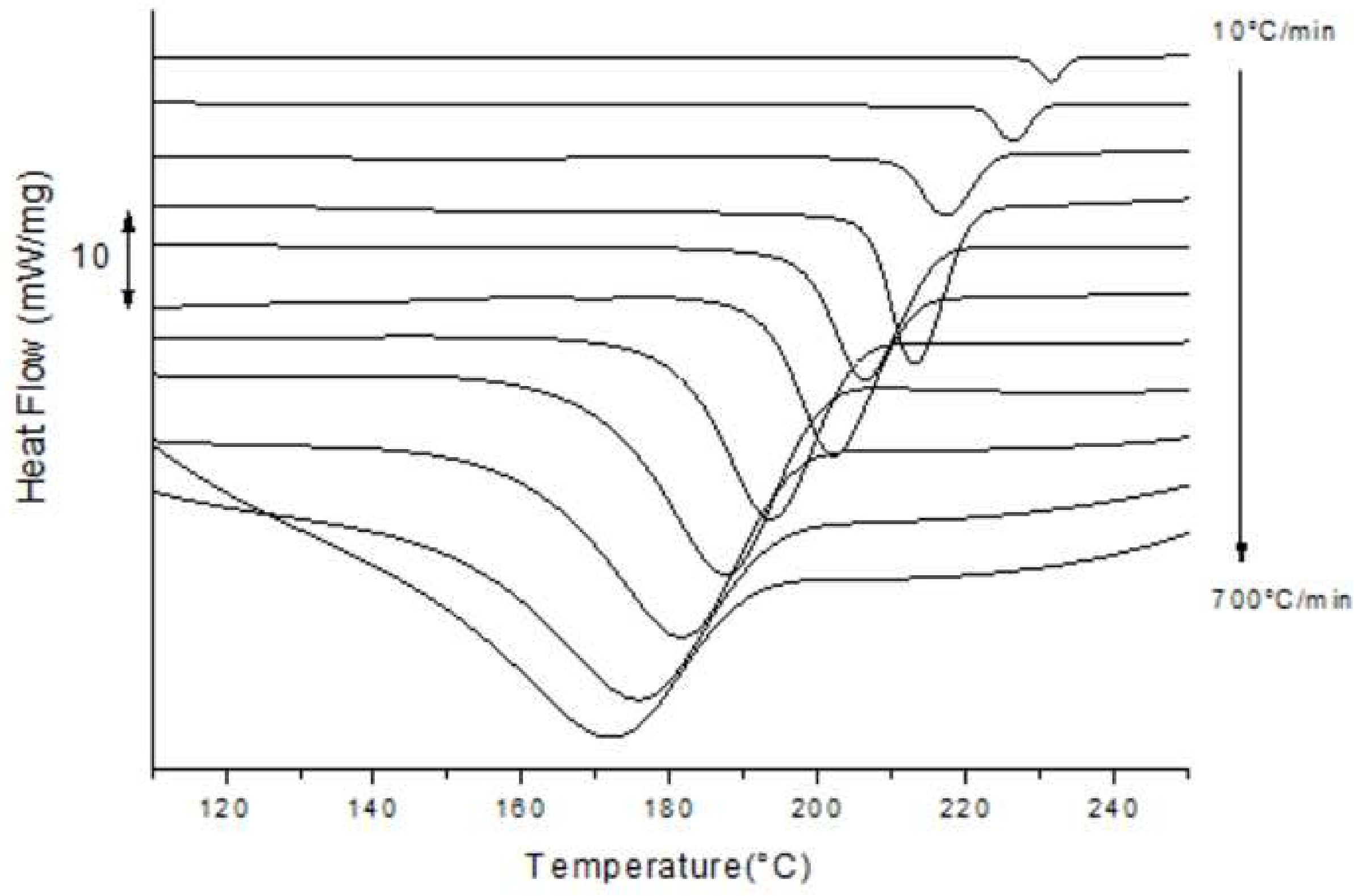




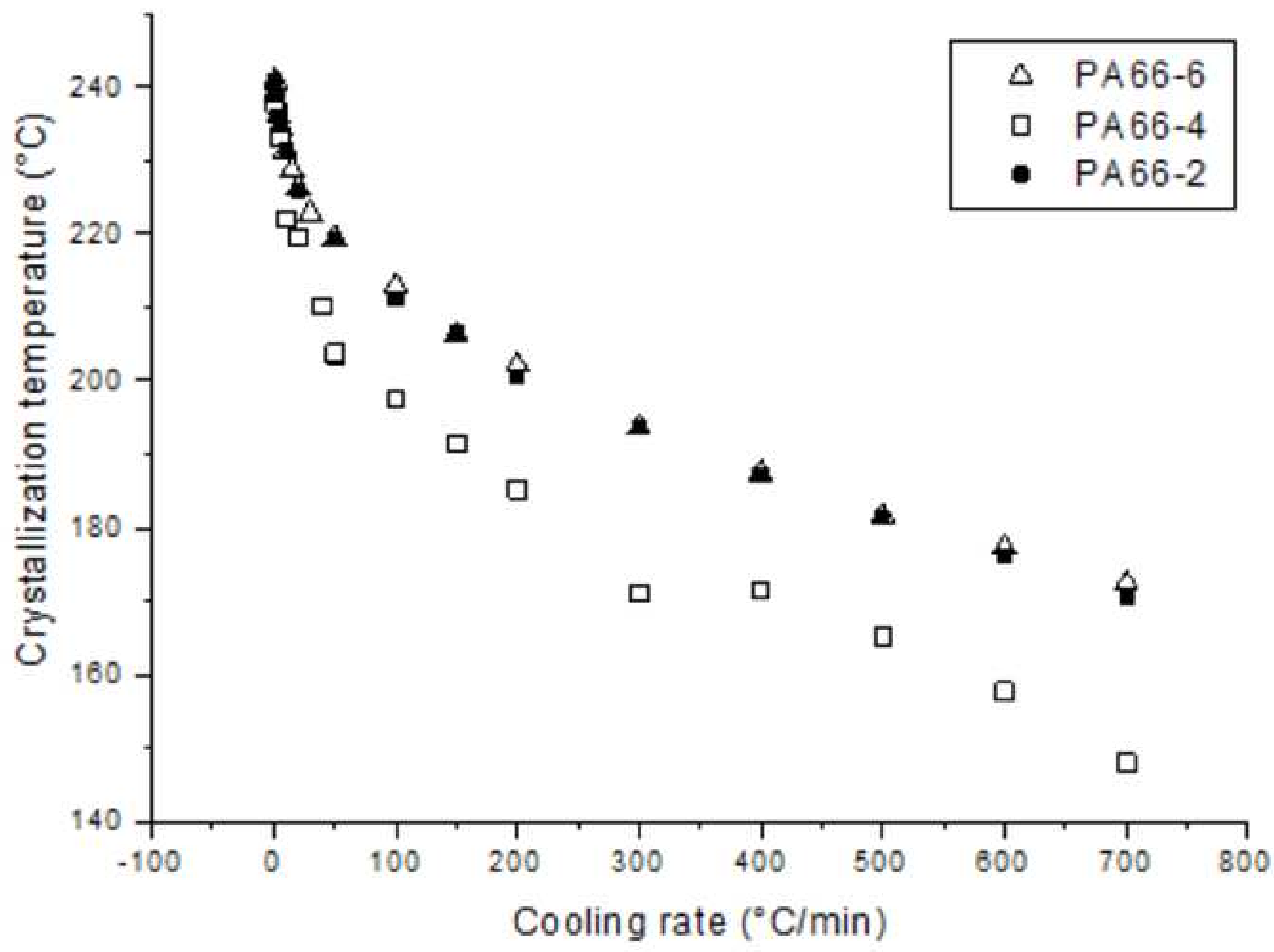




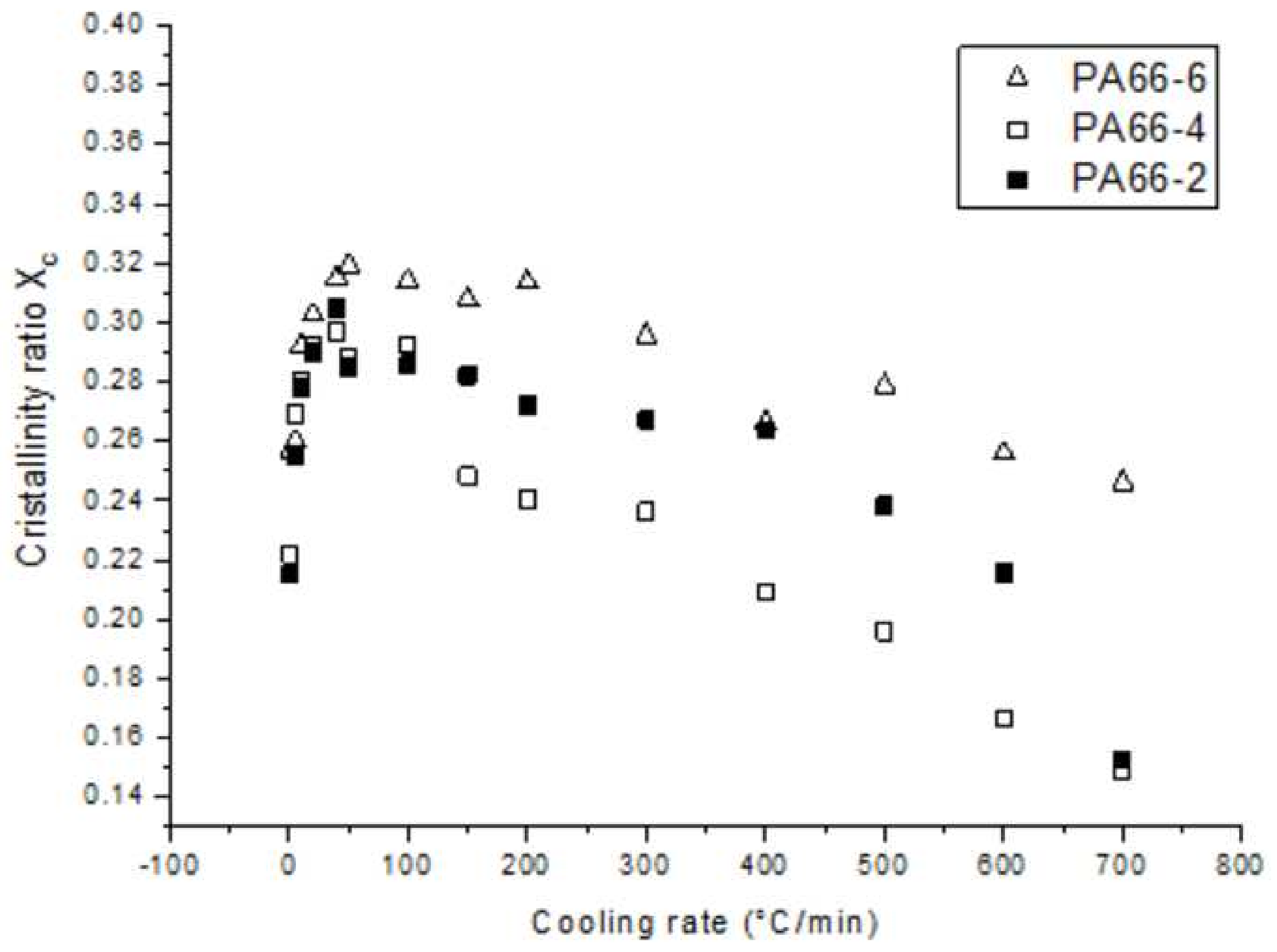




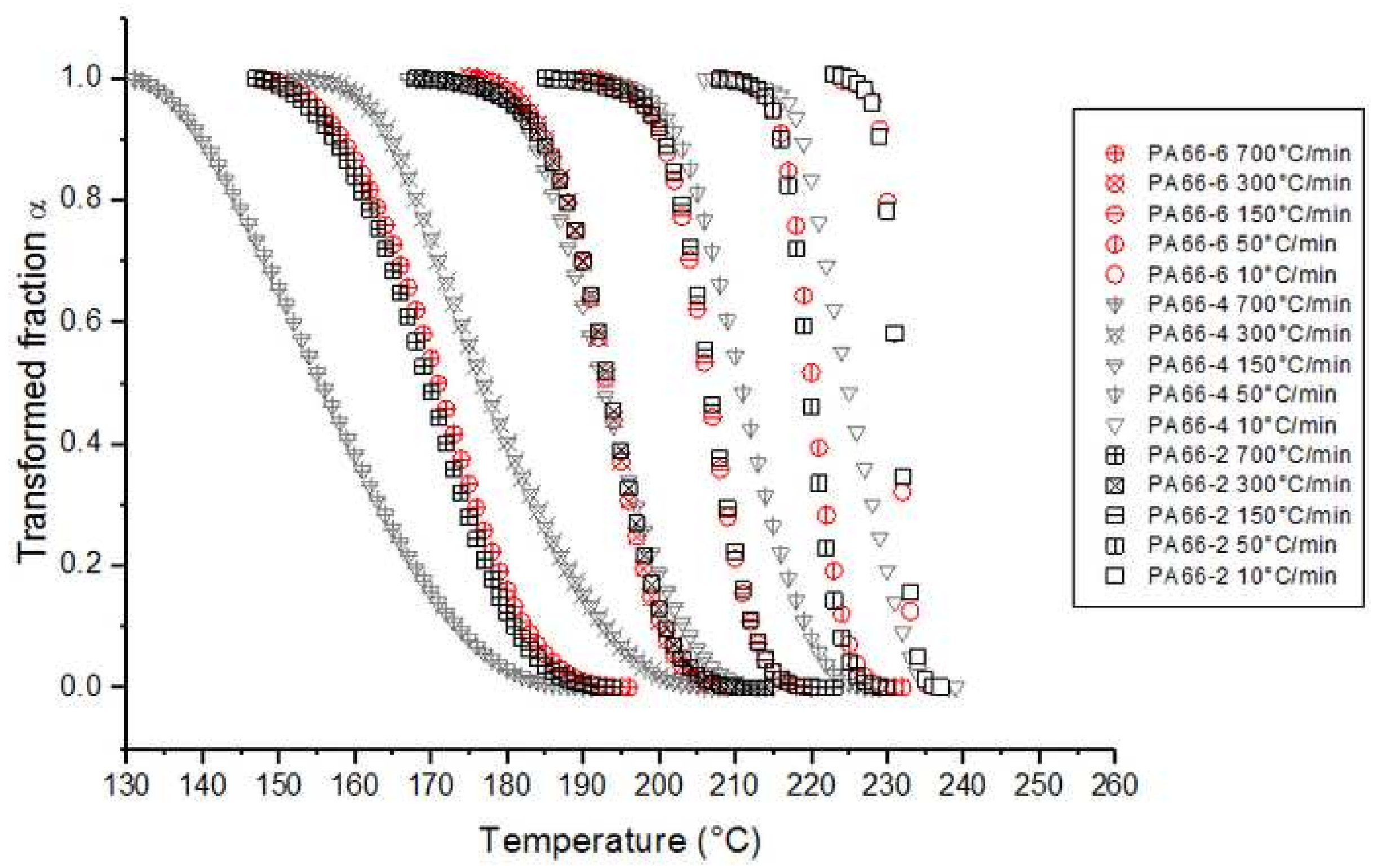




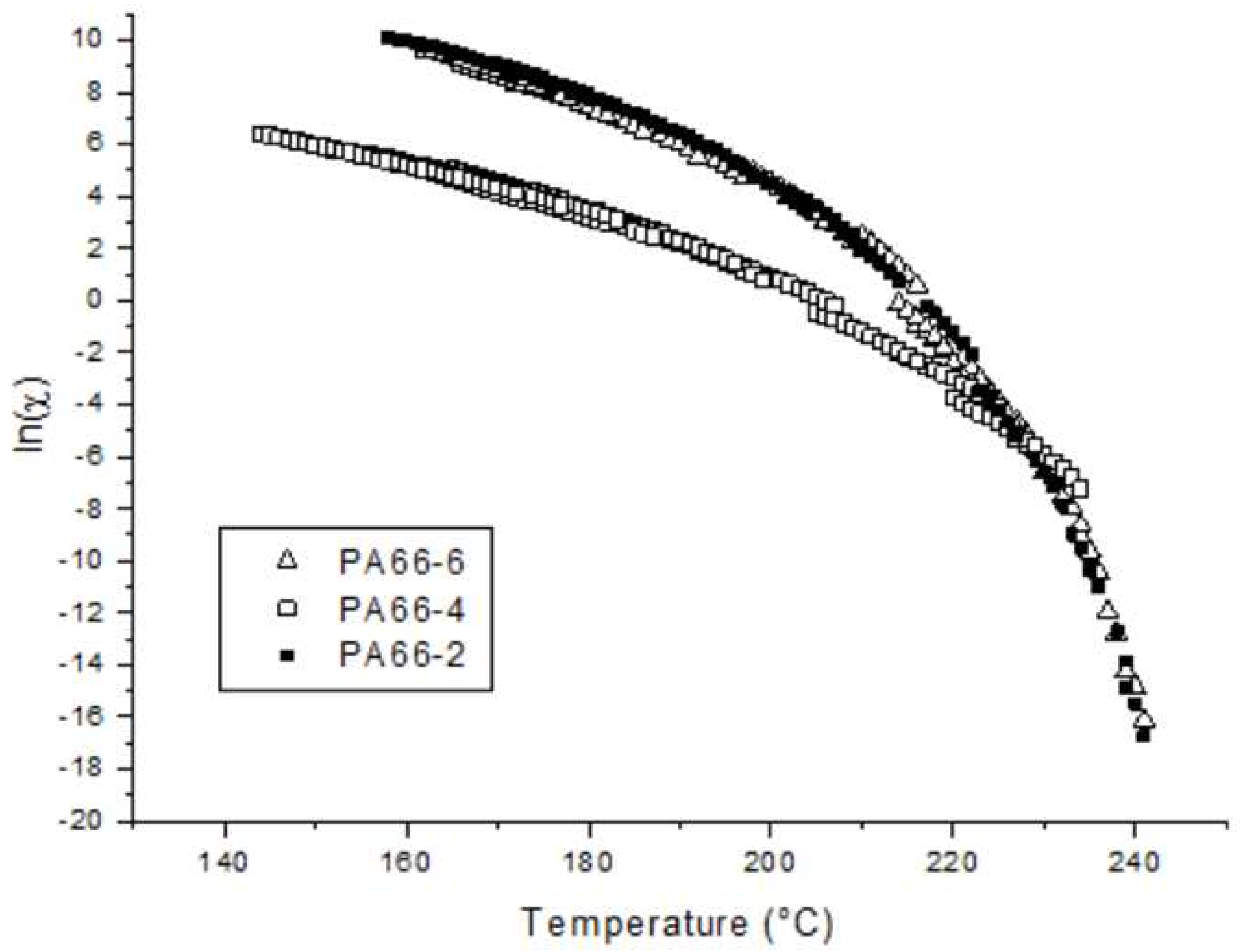




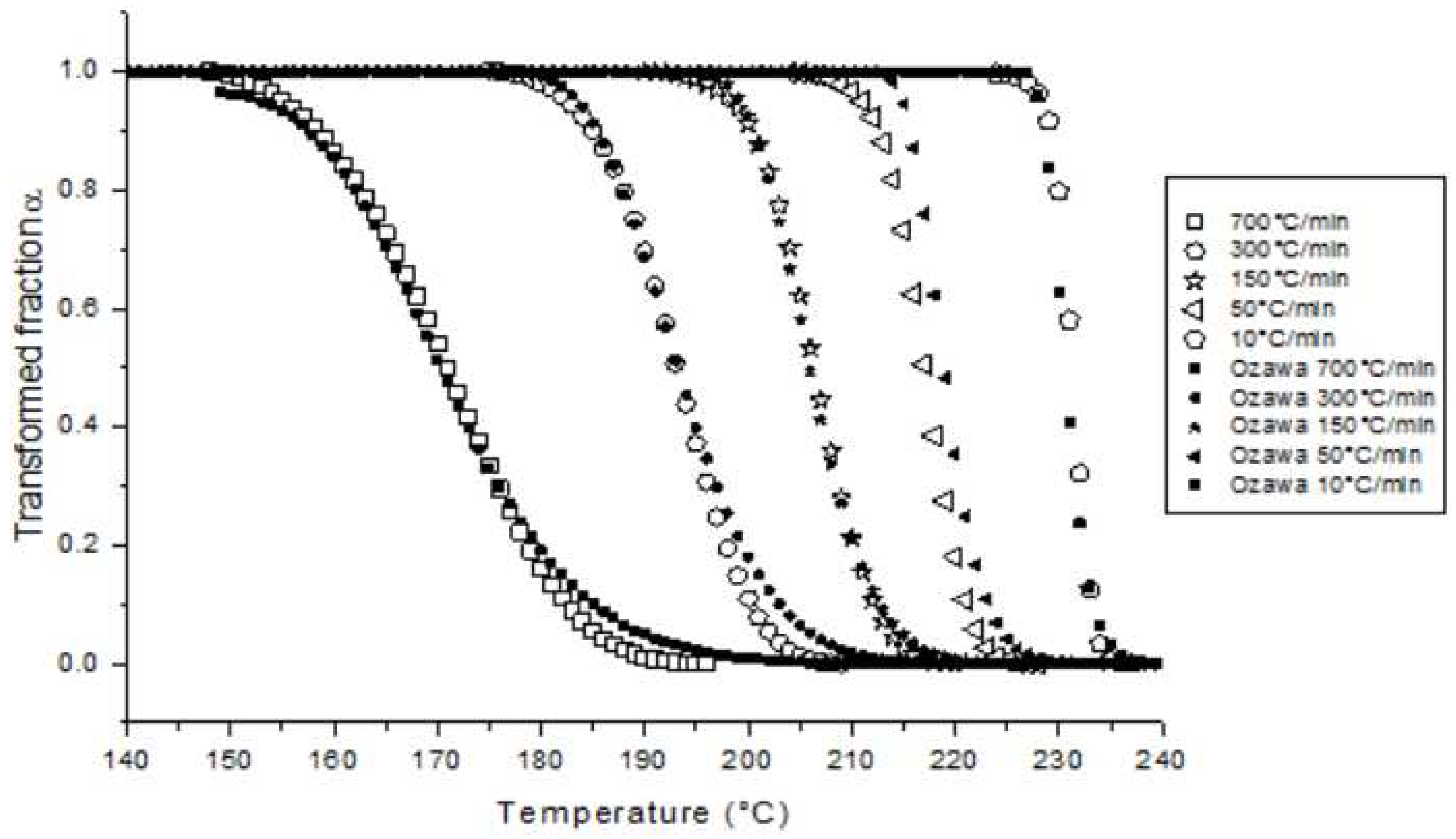




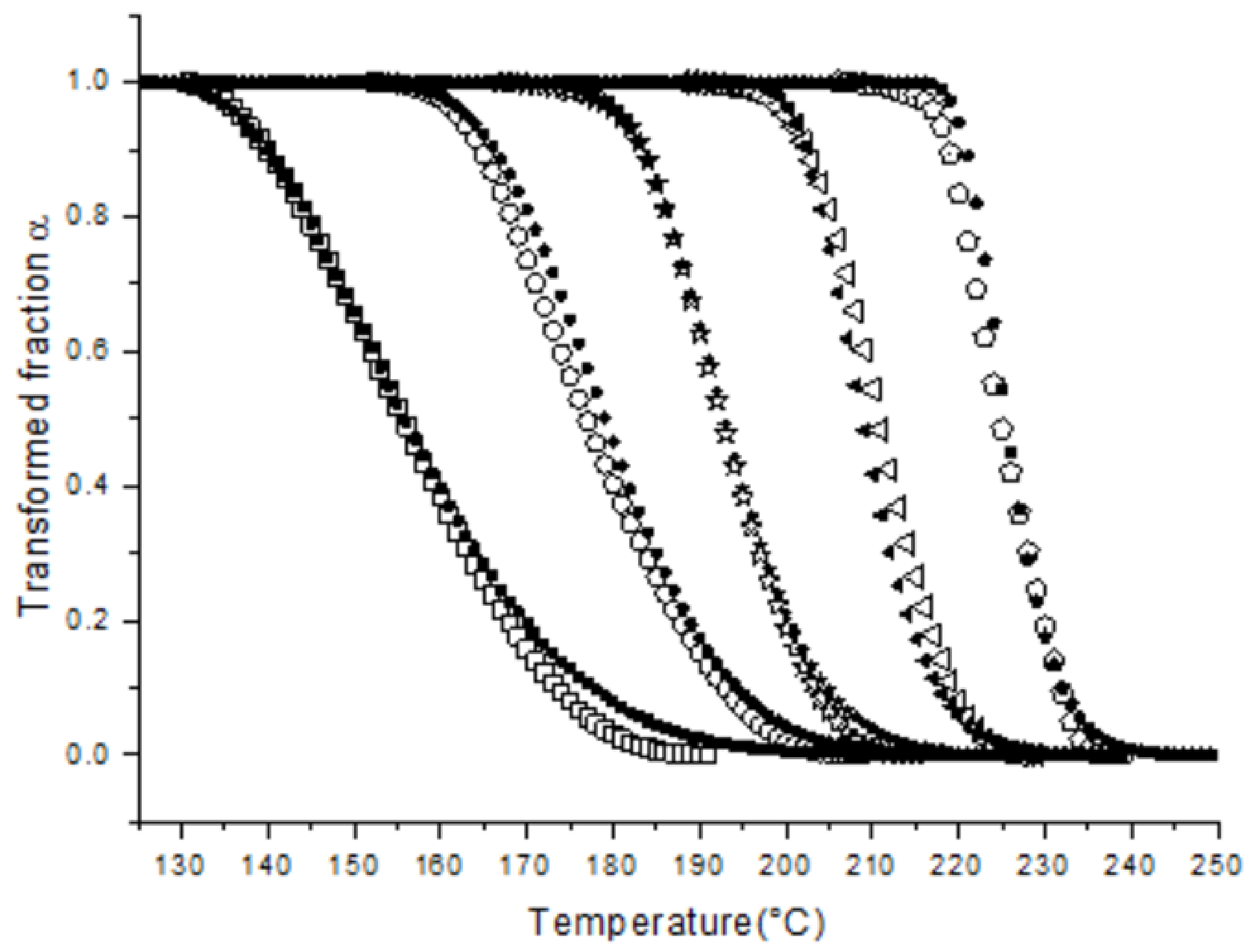



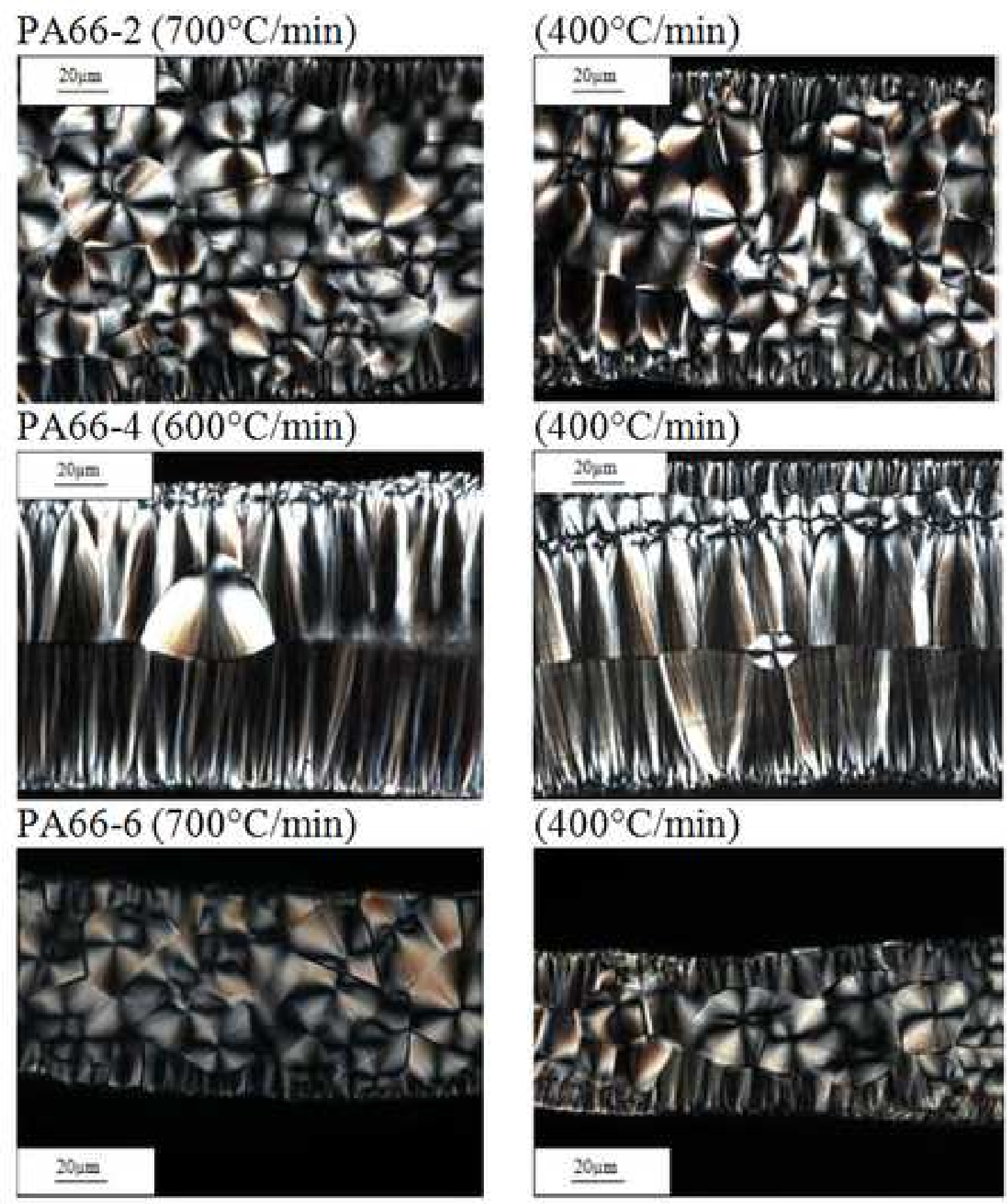

$\left(50^{\circ} \mathrm{C} / \mathrm{min}\right)$

$\left(400^{\circ} \mathrm{C} / \mathrm{min}\right)$

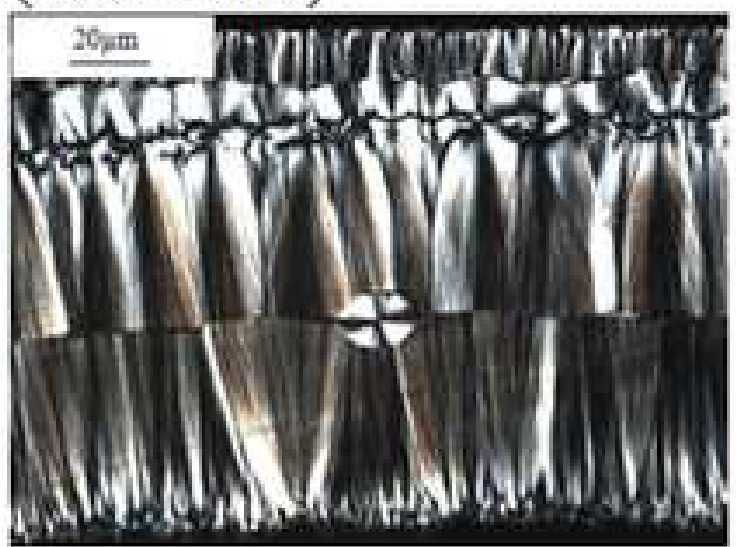

$\left(400^{\circ} \mathrm{C} / \mathrm{min}\right)$
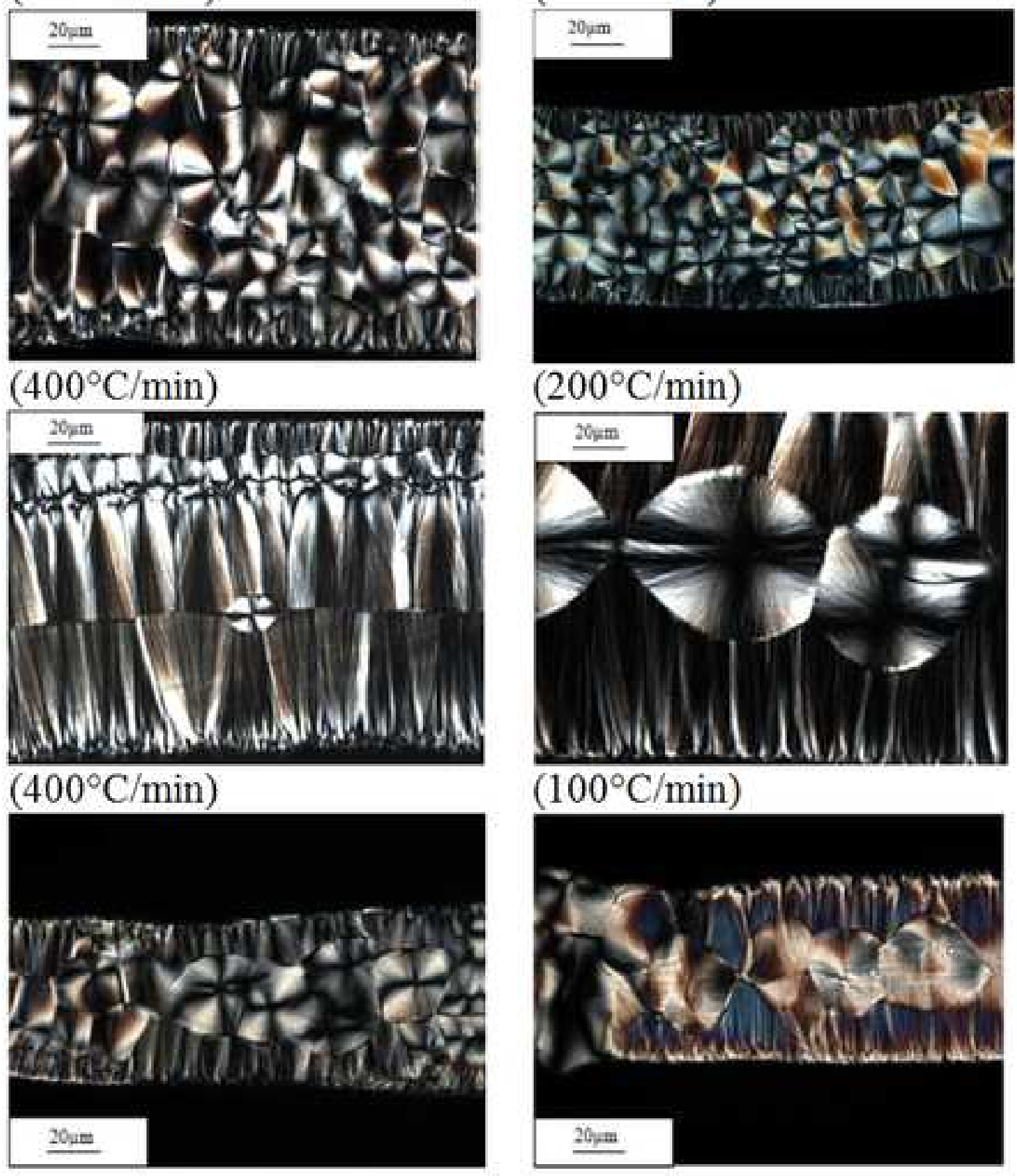

\section{$\left(200^{\circ} \mathrm{C} / \mathrm{min}\right)$}

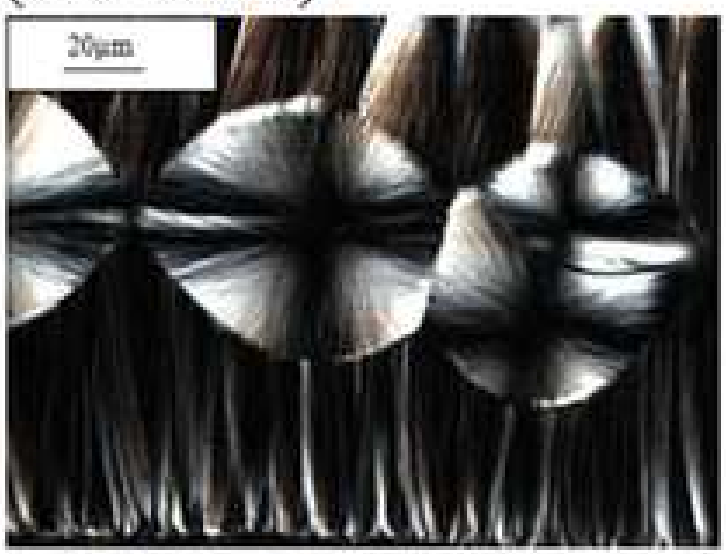
$\left(100^{\circ} \mathrm{C} / \mathrm{min}\right)$

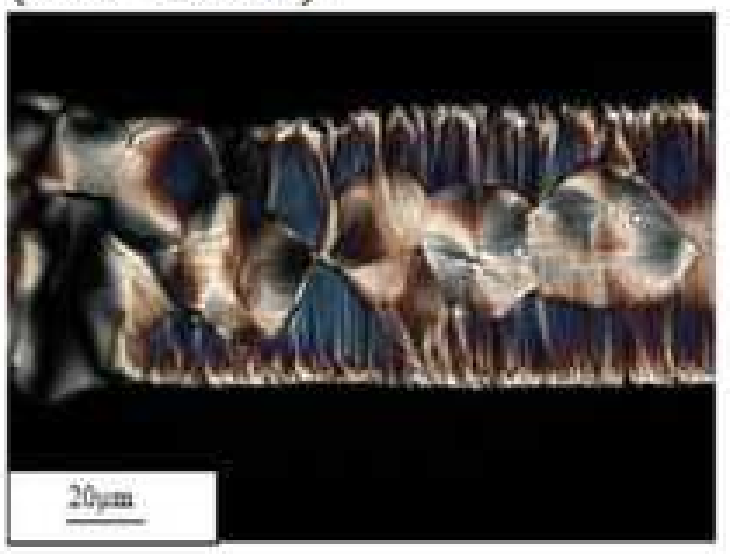




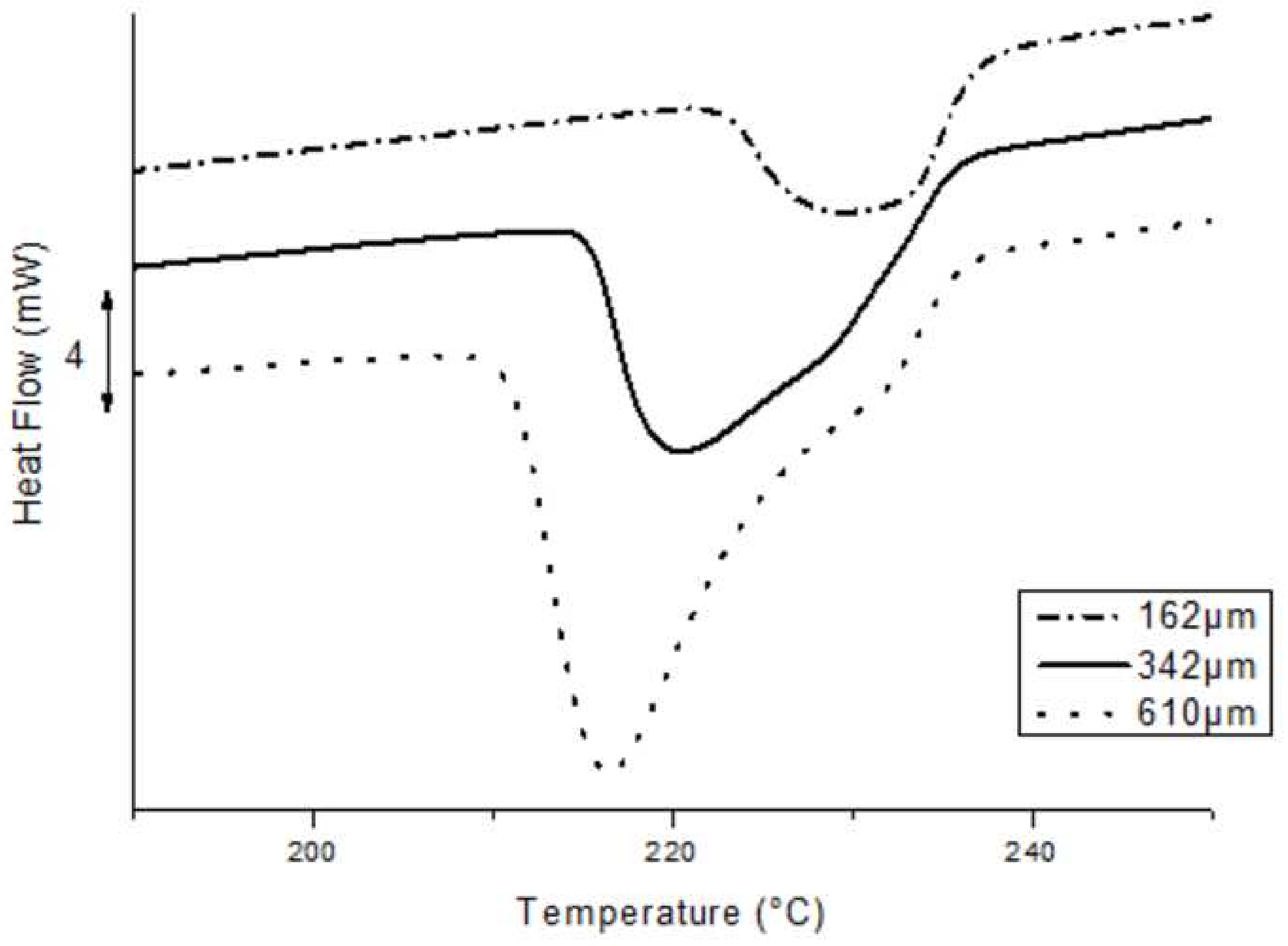


$100 \mu \mathrm{m}$

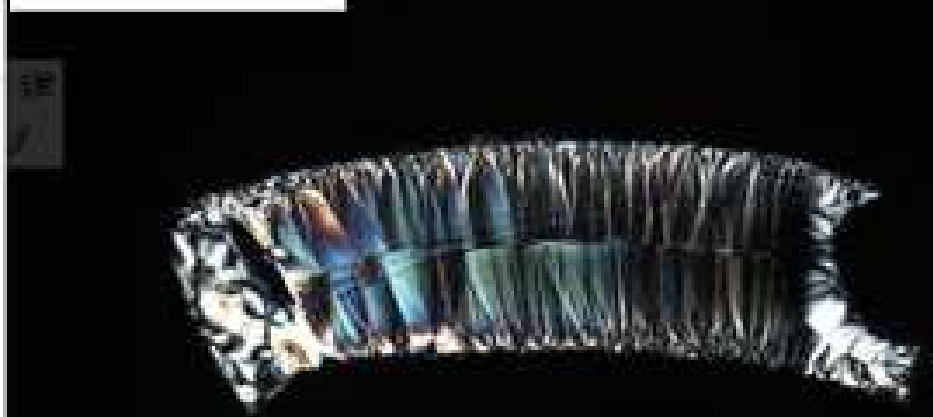

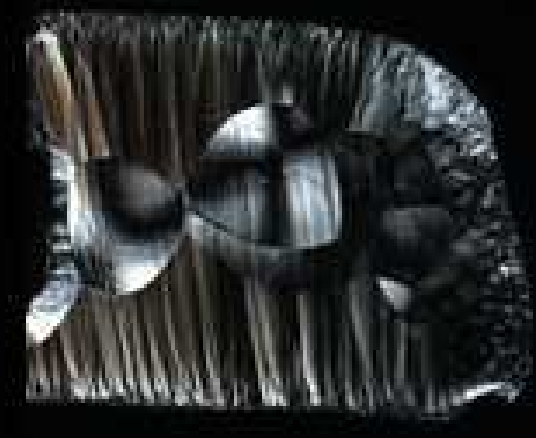

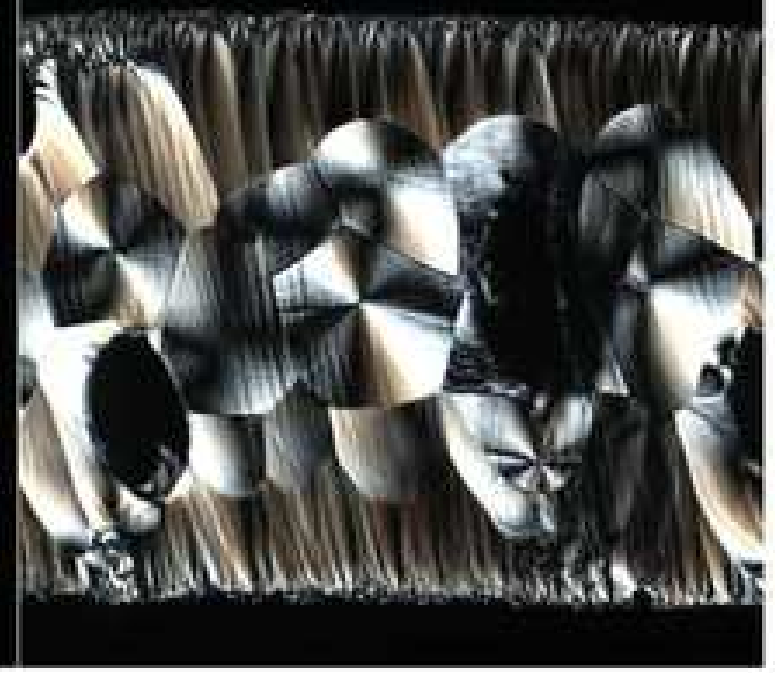




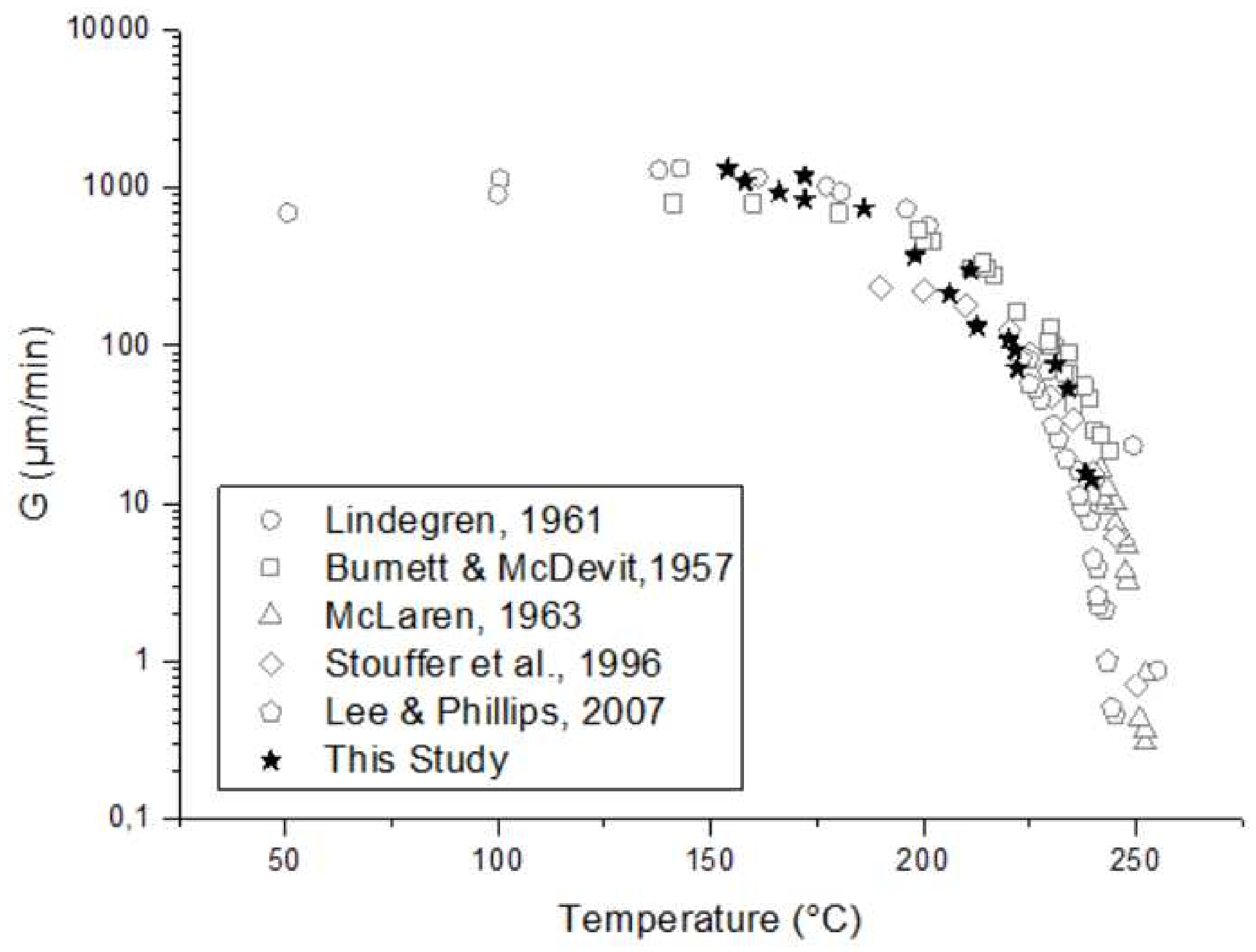




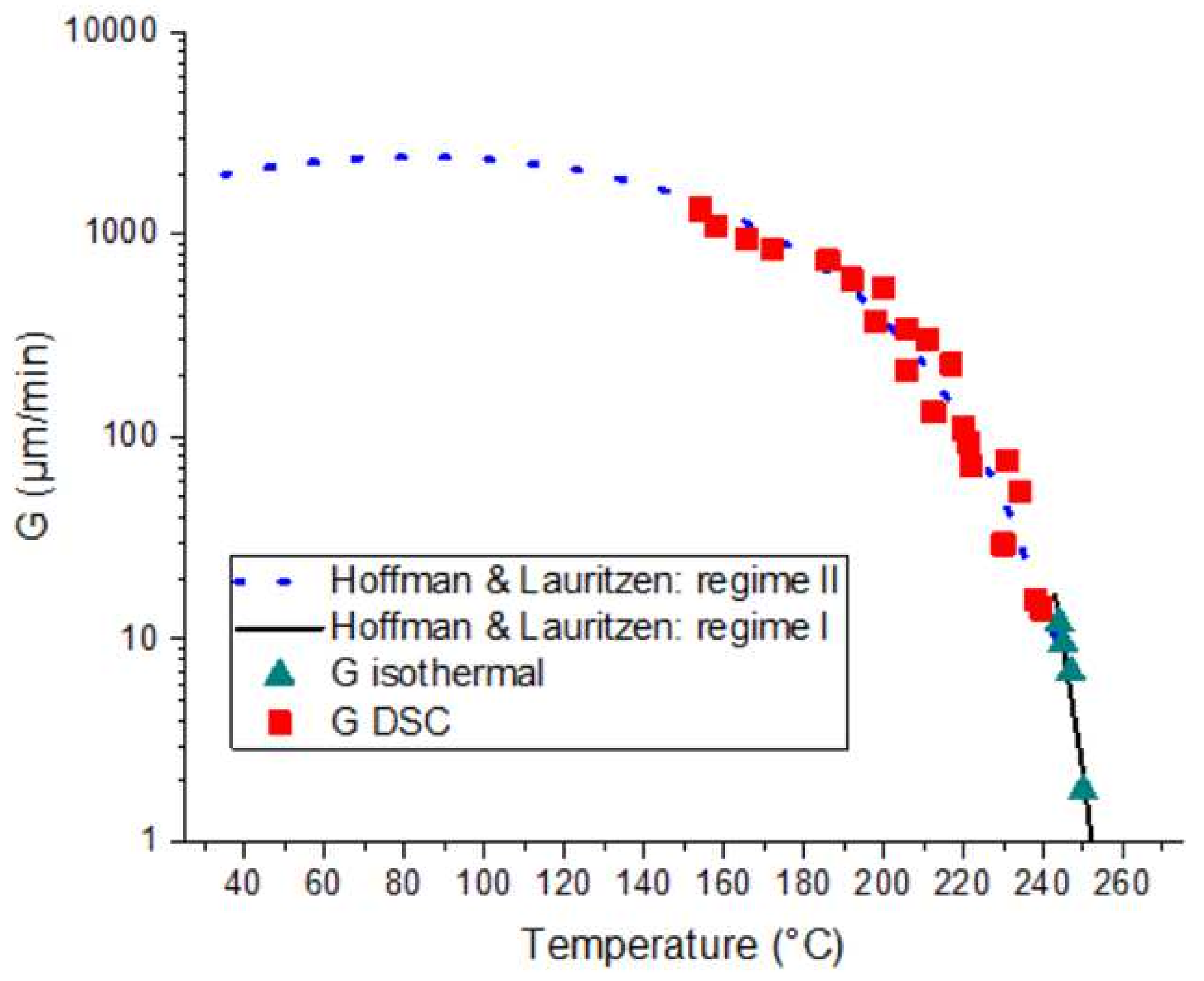




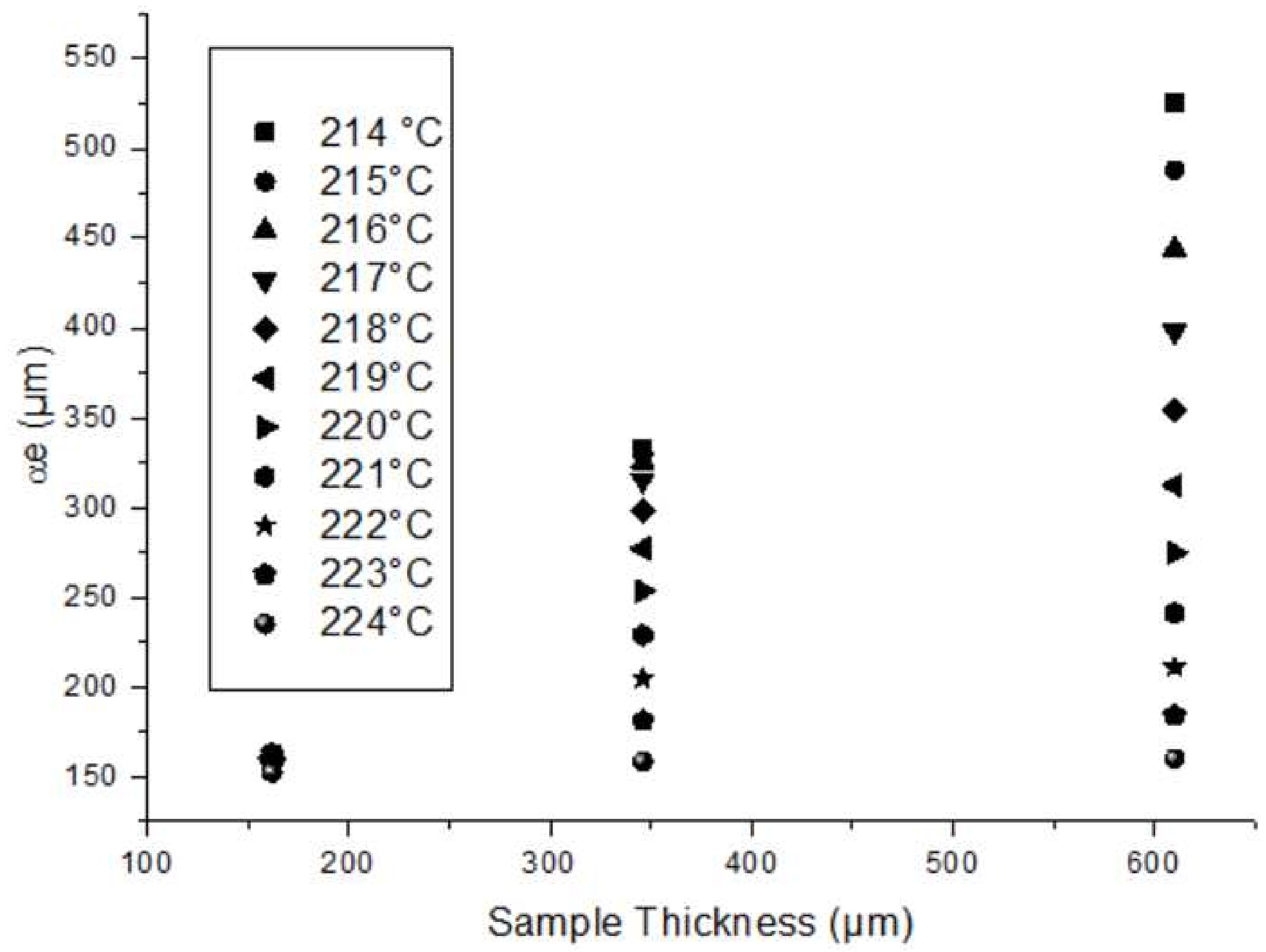




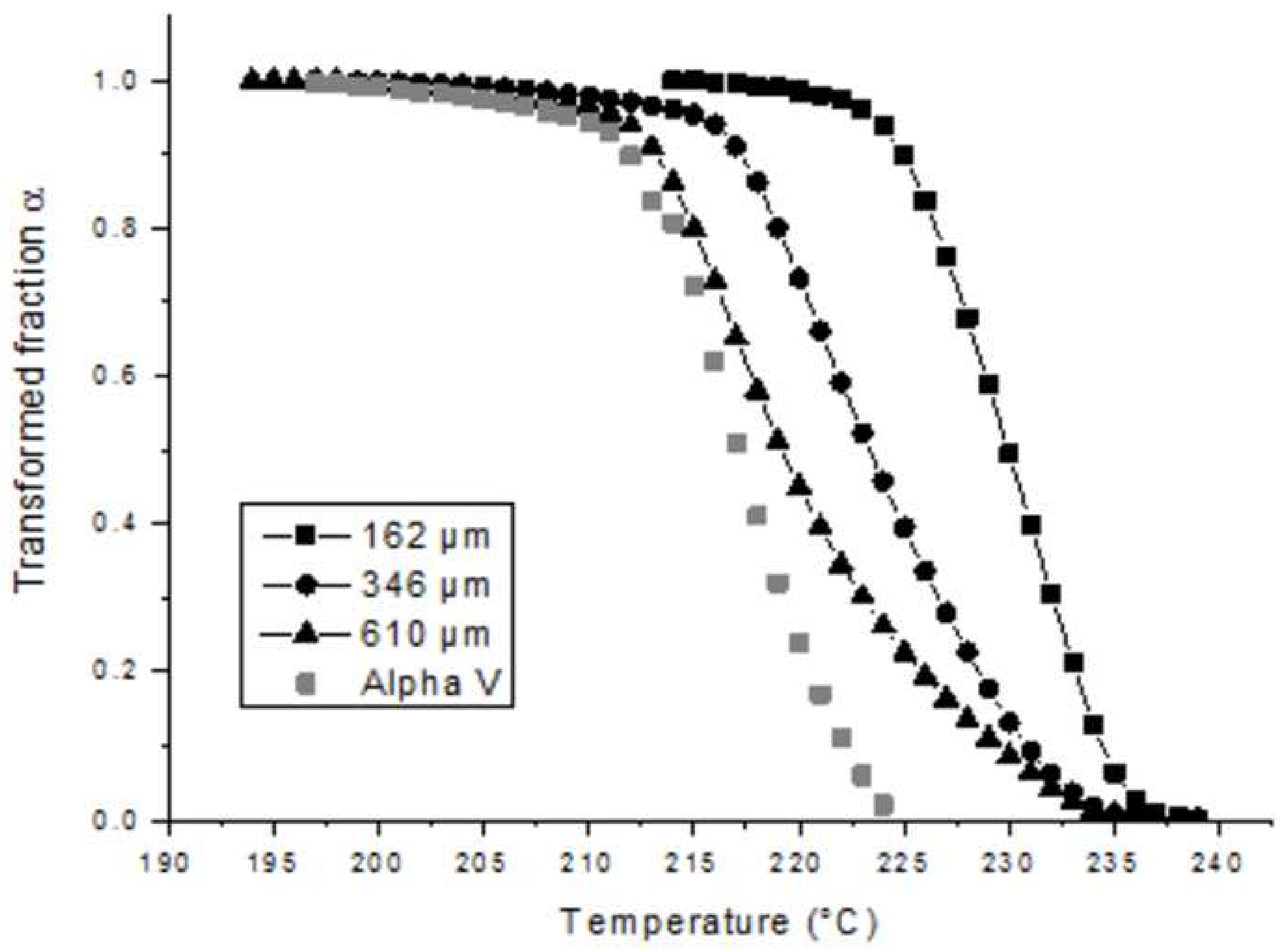




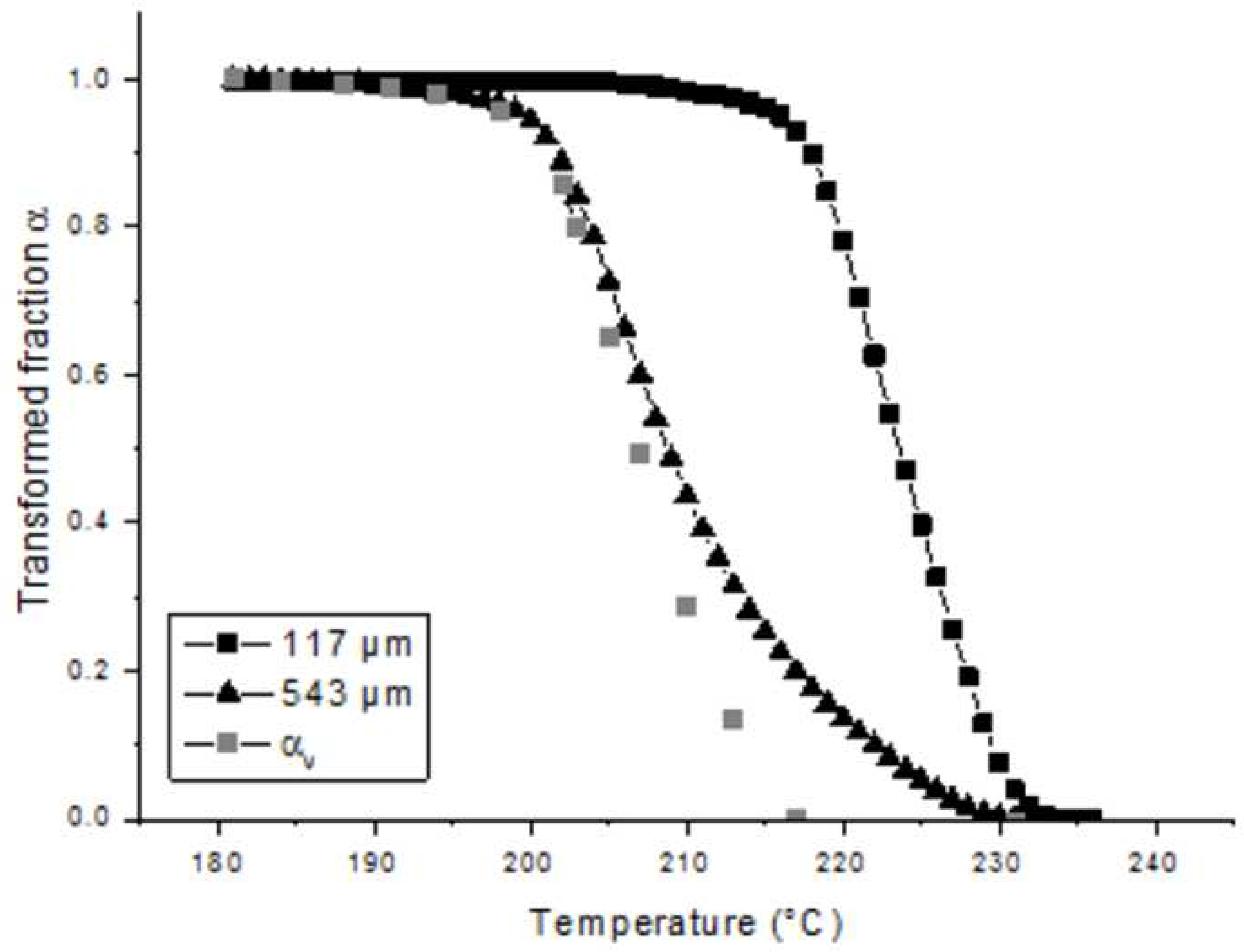




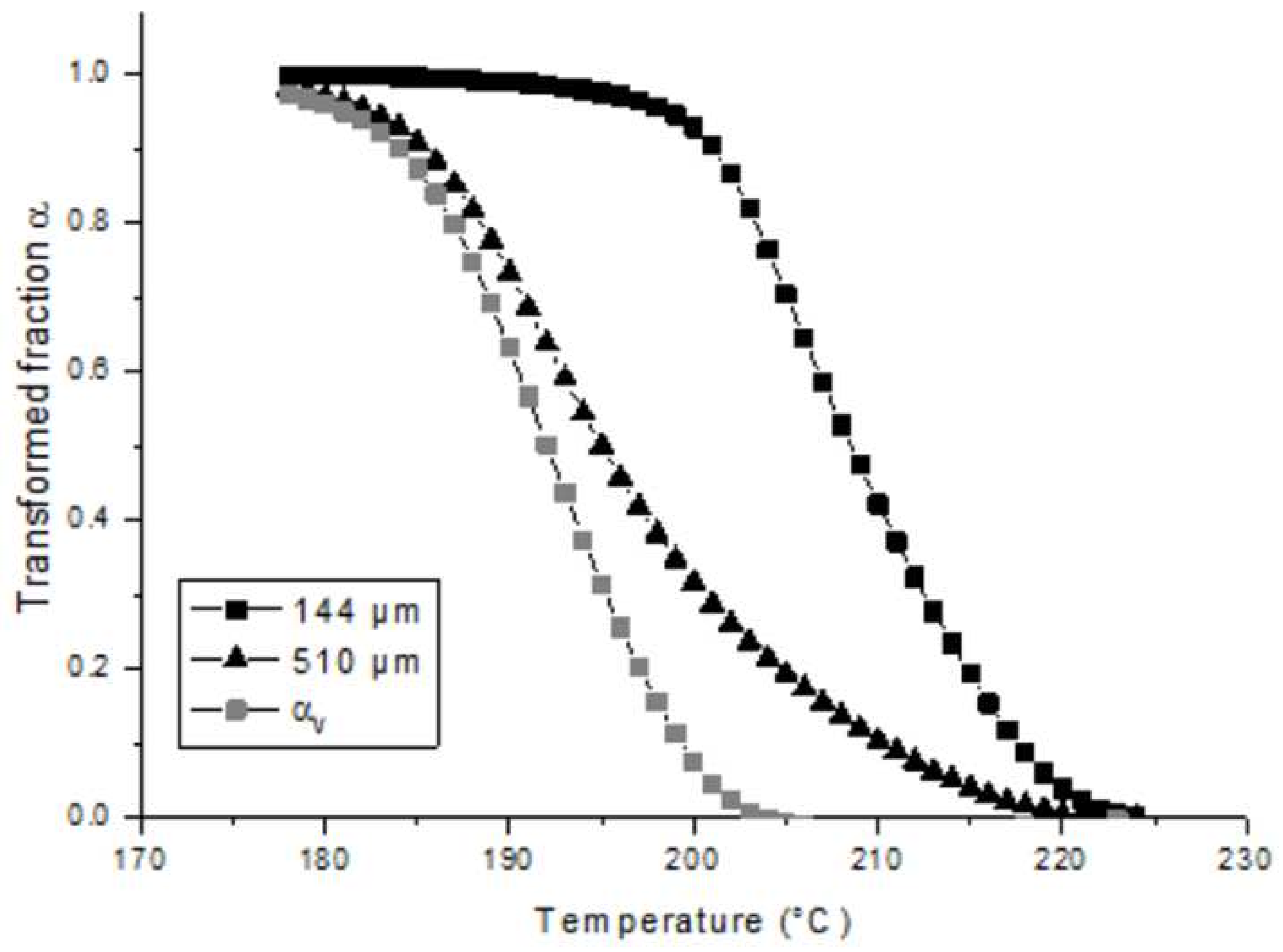




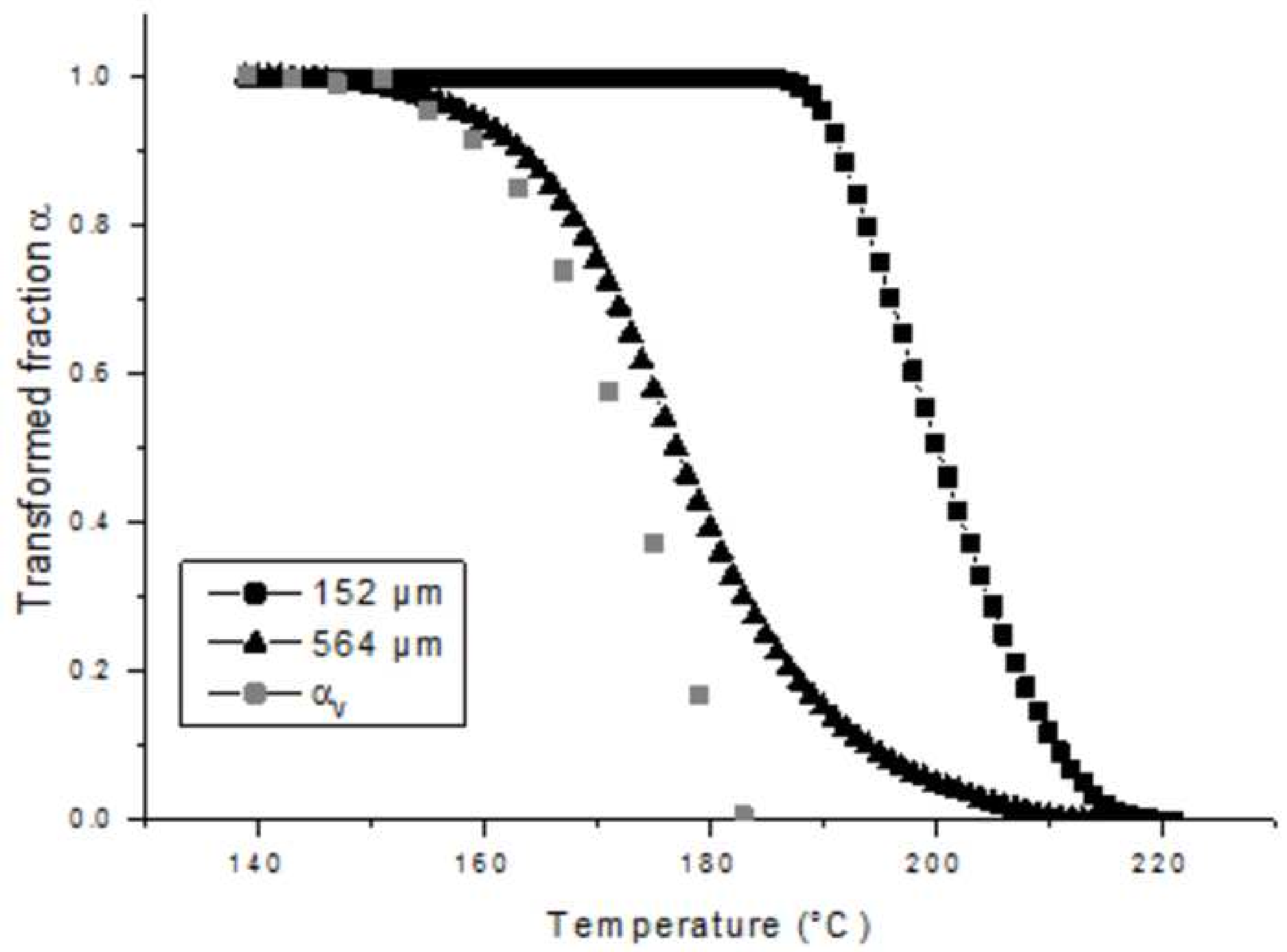




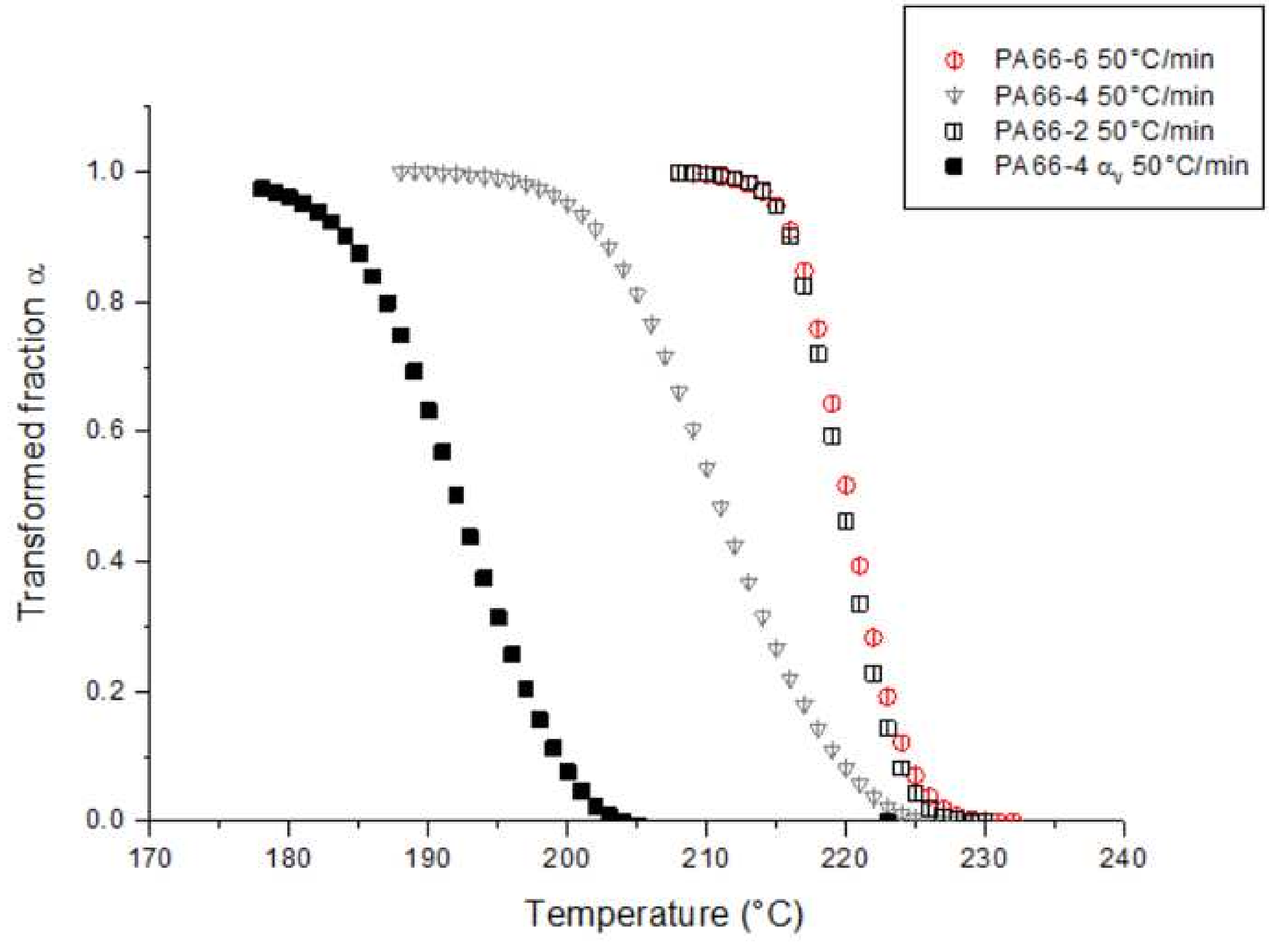

(1) PA66-6 $50^{\circ} \mathrm{C} / \mathrm{min}$

$\forall$ PA $66-450^{\circ} \mathrm{C} / \mathrm{min}$

(1) PA66-2 $50^{\circ} \mathrm{C} / \mathrm{min}$

Click here to access/download;Figure;Fig. 16..tif $\underline{\underline{\underline{ }}}$ 
This paper studied the competition between spherulitic crystallization and transcrystalliny in polyamide 66 by combining optical microscopy and DSC. The influence of transcrystallinity on overall crystallization kinetics is analyzed. In the case of intense transcrystallinity, an original method for growth-rate measurements is applied. Its results compare well with those given by other methods and with literature data. Finally, the "intrinsic" crystallization kinetics of the polymer, i.e., not disturbed by transcrystallinity, is determined.

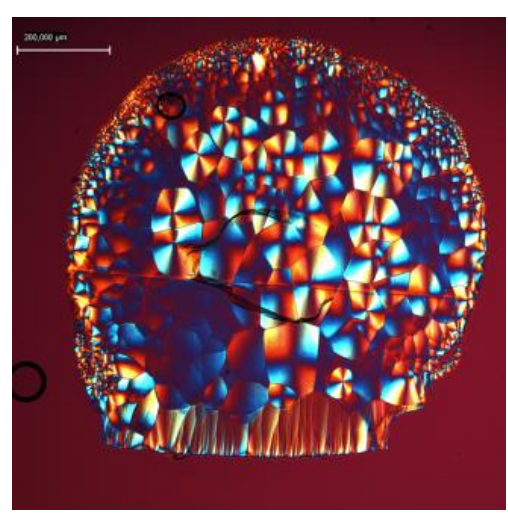

